\title{
1. Discrete Element Modeling of Particle-Based Additive Manufacturing Processes
}

\author{
John Steuben ${ }^{1}$, Athanasios Iliopoulos ${ }^{2}$, John Michopoulos ${ }^{1, *}$
}

\section{Abstract}

A critical element for the design, characterization, and certification of materials and products produced by additive manufacturing processes is the ability to accurately and efficiently model the associated materials and processes. This is necessary for tailoring these processes to endow the associated products with proper geometrical and functional features. In an effort to address these needs in a computationally elegant and at the same time physically realistic manner, this paper presents the development of a methodology for simulating particle-based additive manufacturing processes which employs the Discrete Element Method (DEM). The details of the DEM-based methodology are presented first and the approach is demonstrated on a pair of test problems involving laser sintering of metal powders. The paper concludes with a discussion on how this approach may be generalized to broader classes of additive manufacturing systems, and details are given regarding future work which must be accomplished in order to further develop the present methodology.

5 Keywords: Additive Manufacturing; Layered Manufacturing; Rapid

6 Prototyping; Particle Methods; Discrete Element Methods; Laser Sintering;

7 Laser Accretion; Selective Laser Sintering; Selective Laser Melting; Direct

8 Metal Laser Sintering; Laser Cladding; Laser Engineered Net Shaping;

9 Electron Beam Melting; Direct Metal Deposition; Granular Dynamics; Powder

10 Metallurgy; Multiphysics; Heat Transfer;

\footnotetext{
*Please address correspondance to John Michopoulos, john.michopoulos@nrl.navy.mil

${ }^{1}$ Computational Multiphysics Systems Lab, Center of Computational Materials Science, Naval Research Laboratory, United States

${ }^{2}$ Computational Materials Science Center, George Mason University, resident at Computational Multiphysics Systems Lab, Naval Research Laboratory, United States

Preprint submitted to CMAME

November 4, 2015
}

(C) 2016. This manuscript version is made available under the Elsevier user license http://www.elsevier.com/open-access/userlicense/1.0/ 


\section{Introduction}

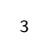

4

6

Additive manufacturing (AM), also known as layered manufacturing or less formally as 3D printing, is an increasingly important technology for the production of a wide variety of components and structures. It consists of a broad family of fabrication techniques which are characterized by successive additions of material to a domain, as opposed to the repeated subtractions which are employed by most traditional fabrication technologies [1]. Recent years have seen a surge of interest in additive manufacturing technology from a broad number of engineering and manufacturing disciplines. This interest is primarily driven by the relative relaxation of geometric constraints provided by additive manufacturing methods; geometries which are difficult or impossible to produce by conventional means are often readily achievable. Additionally, the possibility of producing customized, low volume, or otherwise economically infeasible products [2] has stimulated much interest in the field.

Many of the most prevalent additive manufacturing technologies available at present are based on powders and the challenges associated with their modelling and simulation have been discussed elsewhere [3]. Typically, feedstock material in the form of a fine powder is introduced into the fabrication domain, followed by the directed application of energy to consolidate the powder into some solid form (e.g. sintering or melting). A conceptual example of such powder-based additive manufacturing is shown in Figure 1. While this methodology allows the production of geometrically complex components ranging from biomedical implants [4] to turbine blades [5], it introduces considerable complexity and uncertainty with respect to the material properties and associated functional response.

[Figure 1 about here.]

Because additive manufacturing processes operate in a spatially and temporally varying fashion, the material systems produced by these means can be significantly non-homogeneous, anisotropic, and nonlinear [6]. As a result, 
additively manufactured products are beset by quality issues including dimensional errors, delamination of layers, porosity and poor or indeterminate material properties [7]. These issues are a substantial obstacle to the widespread adoption of additive manufacturing, and often preclude the use of this technology in performance-critical applications [7]. In order to address these issues, and enable the development of design, characterization, and certification tools, it is necessary to develop a methodology for the accurate simulation of powder-based additive manufacturing processes and the resultant products.

In contrast to employing the numerical solution of the multiphysics partial differential equations derived by first principles and the associated conservation laws [3], in this paper we approach the challenge of simulating powder-based additive manufacturing processes by use of the Discrete Element Method (DEM) [8]. In particular, the principal goal of this research is to demonstrate the feasibility of simulating powder-based additive manufacturing processes using the DEM, even under a relatively simple set of physical approximations and assumptions. The DEM is a numerical approximation method for mechanics which is based on an interacting system of particles. These particles interact in a Newtonian fashion, conduct heat transfer, respond to external fields, and may form or break inter-particle bonds. By utilizing these physics encapsulated by the DEM, we enable a simulation which captures the granular dynamics, energy application, and consolidation aspects of powder-based additive manufacturing.

The history and background material regarding powder-based additive manufacturing is the subject of Section 2, and a similar study of the Discrete Element Method is found in Section 3. From this basis, we develop a general DEM formulation for powder-based additive manufacturing problems in Section 4. The details of this algorithm, such as collision detection, consolidation via bond formation, and post-processing are discussed in depth. In Section 5 we discuss the method by which this general formulation may be applied to a specific AM technology, in this case Selective Laser Sintering (SLS). Example results from two SLS test problems are then given in Section 6. The concluding Section 7 presents the discussion of the results achieved, methods for generalizing the present work 
1 to AM technologies that are not powder based, and other advancements which are necessary to further improve the DEM simulation methodology.

\section{Powder-Based Additive Manufacturing}

$4 \quad$ Powder-based additive manufacturing is based on the concept of fusing fine 5 granules of material using directed energy deposition. This is distinct from 6 processes such as stereolithography [9] or Fused Deposition Modeling (FDM)

$7 \quad[10]$ which use liquid or bulk solid feedstocks (respectively). Research towards 8 powder-based additive manufacturing dates back to the late 1970's [11], with 9 commercial processes and systems emerging in the late 1980's and early 1990's 10 [12]. Numerous processes have been developed, first using polymers and more 11 lately encompassing metals, ceramics, and other materials. Details on some of 12 the most common techniques currently in use are described below. Generally, 13 the standard terminology given by the ASTM standard 2792 [13] is employed 14 hereafter.

SLS is the oldest of the powder-based additive manufacturing technologies and was developed in the late 1980's at the University of Texas [14]. This process distributes a powder feedstock, usually a polymer, in a thin uniform layer across the top surface of the build domain. A laser energy source is then used to selectively fuse the powder via sintering. The build domain is lowered and the process is repeated until the specified object has been completed. This process typically occurs under an inert atmosphere in order to prevent oxidizing reactions. Further details on SLS can be found in [15-17]. While SLS most commonly employs polymer feedstocks, metal powders are also used. In cases where metals are used, the term Direct Metal Laser Sintering (DMLS) is commonly employed.

DMLS is a direct descendant of SLS $[18,19]$ and varies from that process only in the fine details which differentiate the bonding mechanisms of metals 28 and polymers [20]. Stainless steels [21], titanium alloys [22, 23], copper alloys 29 [24], nickel alloys [25], aluminum alloys [26], and various other materials have so been widely used with the DMLS process. 
Selective Laser Melting (SLM) operates on a similar principle to SLS and DMLS, but employs higher energy densities in order to selectively heat the feedstock powder above the liquidus temperature [27]. By doing so, lower void fractions and higher densities are achieved in manufactured components. However, because of the higher energy density and the complex behaviors of the molten pool, control of the process is more difficult and deleterious phenomena such as thermal stresses emerge [28]. Work in SLM has primarily been focused on iron and steel feedstocks [29], but some work has been done with nickel superalloys [30].

Electron Beam Melting (EBM), sometimes known as Selective Electron Beam Melting (SEBM) is closely related to SLM. In EBM energy input is accomplished by means of an electron beam, which is directed using magnetic lenses. Unlike the technologies described previously, EBM occurs in a vacuum environment and the feedstock materials are preheated to a greater extent. A comparative review of EBM and SLM is offered by [31]. EBM permits the use of a very broad range of materials; steels [32] and titanium [33] are common, but exotic materials such as niobium [34] and beryllium have also fabricated by means of EBM.

Direct Metal Deposition (DMD) [35, 36] and Laser Engineered Net Shaping (LENS) [37, 38] are processes for powder-based manufacturing which differ substantially from the examples given earlier. Instead of fusing stationary beds of powder, DMD and LENS processes actively inject or "jet" the feedstock material into a melt pool produced by a laser thus combining mass with energy deposition. Typically the laser optics and particle jet nozzles are mounted concentrically on the end of a robotic manipulator. This allows for the construction of very large components, as well as the deposition of material onto existing components in repair applications [39].

The combination of processes and materials which are available using powderbased additive manufacturing has led to widespread use of this technology across many industries [7]. In both the case of powder bed methods such as SLS and powder jet methods such as DMD it is clear that the mechanics of granular 
1 systems play a large role in the AM process. A methodology well-suited for the 2 study of such systems is introduced in the next section.

\section{The Discrete Element Method}

The Discrete Element Method (DEM) is a numerical method for simulating the behavior of both continuous and discontinuous material systems. DEM simulations are based on the Newtonian interactions of a system of particles. These particles are imbued with constitutive behaviors including contact/collision models, heat transfer models, the ability to form and break interparticle bonds, and respond to external fields. While first discussed in [40], a modern general reference on the DEM can be found in [8]. Other, more applied, overviews are given by $[41,42]$. The interested reader will find that a great deal of the research on the DEM is found in the geomechanics and rock mechanics literature, owing to this method's usefulness in problems of brittle fracture (e.g. $[43,44])$. Other common areas of application are the study of granular flows [45], ball mills [46], and ballasts [47].

\subsection{Formulation}

A DEM simulation contains a number of abitrarily-shaped particles, a subset of which may be "bonded," or attached to each other. To the extent that they contact each other both bonded and unbonded particles participate in Newtonian collisions which produce reaction forces which in turn propagate into changes in particle velocity and position. A schematic overview of the associated concepts from the perspective of interacting particles is shown in Figure 2. While this figure depicts spherical particles, it important to note that this approximation is not generally applicable. Because contact stiffness is a function of local curvature, and contacts between aspheric particles will result in tangential and moment forces, this approximation must be applied with care. The major conceptual elements of a general DEM implementation are as follows:

[Figure 2 about here.] 
1 1. Collision detection. Generally, it is not practicable to exhaustively check 2 every particle in the DEM simulation for contact with all other particles; such a search would be of $\mathcal{O}\left(n^{2}\right)$ complexity where $n$ is the number of particles and ${ }_{4}$ likely greater than $10^{6}$. For this reason, spatial subdivision schemes are used so 5 that collision searching is reduced to local neighborhoods. Common techniques for spatial subdivision include grids [48], octrees [49], and heap sorting [50]. An extensive review of collision detection techniques is given by [51].

2. Inter-particle contact laws. Once particles are determined to be in contact, an appropriate contact relationship that captures the material constitutive response must be applied. The most common form is a force-displacement rela11 tionship with both normal and shear terms, as shown in Figure 2. The simplest 12 formulation in terms of the normal $\left(\mathbf{f}_{n}\right)$ and shear $\left(\mathbf{f}_{s}\right)$ forces has the form

13

$$
\mathbf{f}_{n}=k_{n} \delta \hat{\mathbf{x}}_{n}
$$

$$
\mathbf{f}_{s}=-\mu\left\|\mathbf{f}_{\mathbf{n}}\right\| \frac{\left(\mathbf{v}_{i}-\mathbf{v}_{j}\right)}{\left\|\mathbf{v}_{i}-\mathbf{v}_{j}\right\|}
$$

where $k_{n}$ is a stiffness coefficient, $\mu$ is a friction coefficient, $\delta$ is the interpenetration distance defined in Figure 2 and $\mathbf{v}_{i}, \mathbf{v}_{j}$ are the velocity vectors of the particles participating in the collision and $\hat{\mathbf{x}}_{n}$ is the unit normal vector connecting the particle centers. Many more sophisticated contact models are available and a summary of normal-force models can be found in [52], while a discussion of shear-force models is given by [53]. Among the most frequent augmentations made to the simple model of Equations (1-2) is the inclusion of heat conduction.

3. Boundary condition contact laws. The interacting particles of the DEM typically do not occupy an infinite unbounded space, therefore provisions for boundary conditions must be made. The most common boundary conditions are stationary and moving infinite planes. Testing particles for collision with these planes is trivial and efficient. Periodic boundary conditions, e.g. those employed in [54], are also common. For more complex cases, collision with 
1 triangular meshes is commonly employed, using methods such as that given by 2 [48]. The contact laws applied are not necessarily the same as those for interparticle collisions, and may embody different constitutive behaviors than the particle-particle case.

5 4. Bond formation, deformation, and breakage. An important feature of the 6 DEM is the formation of bonds between particles. This allows the simulation of continuum domains and solid objects. Generally bonds are considered as two or three-dimensional beams, which may support selected combinations of axial, shear, and moment loadings. The use of bonds to capture the long-range properties of materials is discussed in depth by [55]. Once formed, bonds deform under mechanical forces, and break once a failure criterion is reached. This allows the simulation of complex failure modes, such as delamination or fracture [56], in a computationally efficient fashion. This topic is further examined by $[57]$.

15 5. Time step integration. Once the physical influences (e.g. forces, heat fluxes) ${ }_{16}$ on every particle have been determined, it is necessary to propagate these influences motions of the particle system and step the DEM simulation forward in time. An explicit Eulerian $[58,59]$ or Verlet $[60,61]$ time-step integration is most widely used for this purpose. However, recent work has shown that implicit methods are promising in this respect $[59,62]$.

\subsection{Related Methods}

The Discrete Element Method is only one of many particle-based methods which find use in numeric simulations. One widely-used technique closely related to the DEM is Molecular Dynamics (MD) simulation [63]. DEM and MD are broadly similar methodologies, some researchers use the terms interchangeably [64]. However, the DEM is distinct from MD formulations. It supports polydisperse particle systems, non-spherical particles, and particles with rotational degrees of freedom; these features are not commonly found in MD implementations. We have selected DEM as the apropriate simulation methodology for 
additive manufacturing exactly because of these features. In particular the feature of polydispersity makes DEM uiniquely prefereable due to the fact that it enables accurate representation of feedstock powders that are characterized by multiple particle size distributions.

Another widely used particle-like method is Smoothed Particle Hydrodynamics (SPH) [65, 66], although in this case the term 'hydrodynamics' should

be read as 'mechanics.' In SPH, particles are augmented with a spatial 'smoothing length' over which their properties are interpolated by a kernel function. A comprehensive review of SPH may be found in [67]. SPH is most widely used to simulate fluid mechanics [68], but has also been extended to the domain of solids [69]. A thorough comparison of DEM, SPH, and MD methods is given by [70]. Because powder-based additive manufacturing bears a much greater similarity to the DEM formulation than SPH, we have concluded that employing the former represents the most appropriate path forward. However, SPH may be a valuable tool for simulating other AM technologies, especially when used in a hybrid fashion with the DEM (e.g. the method of [71]).

\subsection{DEM for Additive Manufacturing}

The DEM has been widely employed to study granular systems [45, 53], including pressure sintering [53]. However, it has not been employed to study additive manufacturing processes to the same extent. One preliminary example from the literature can be found in [72], however this example focuses on the development of random packings, and does not investigate the physics of the sintering process. From the discussion above it is clear that DEM has great potential in this field. In the next section, we outline an algorithm which leverages the well-suited formulation of the DEM to simulate powder-based additive manufacturing in a physically realistic and computationally efficient fashion.

\section{Methodology}

Based on the review of powder-based additive manufacturing systems presented in the previous section, it is clear that DEM simulations of these pro- 
cesses must perform several tasks in the most computationally efficient manner achievable. These tasks are given in a general form below.

1. The DEM simulation must embody a system of particles with properties corresponding to the feedstock material for the AM process (either polymer or metal). Both the mechanical and thermal responses of the feedstock materials must be accurately modeled. The particle system itself must be expandable, in order to model continuous (as in the case of DMD) or successive distinct (as in the case of SLS) additions of material to the build domain over the course of the additive manufacturing process.

2. The boundary conditions of the simulation must define a domain mimicking that of the additive manufacturing process. In the case of AM processes such as SLS, this ultimately requires the arrangement of particles into uniform flat layers.

3. The DEM simulation must apply heat energy to the particle system in such a fashion that mimics the additive manufacturing process. In practice, the simulation is required to read a computer numeric control (CNC) machine code file, and position a heat source according to the directives of this code.

4. The particle system must react to the application of heat energy by forming bonds, in order to model the consolidation process (sintering or melting) employed by the additive manufacturing technique.

5. An animated visual display of the particle system must be displayed to the user of the simulation.

In order to accomplish these tasks, we developed a general DEM framework for the simulation of additive manufacturing processes. The details regarding different aspects of this framework, the constituent algorithms, and implementation are given in the sections below. Following this, in Section 5, the application of this general framework to a particular additive manufacturing process is given. 


\subsection{Particle System Definition}

In this work we utilize a DEM particle system of $N$ spherical particles occupying a build domain $\Omega \in \mathbb{R}^{3}$ with a boundary $\Gamma$, with Cartesian spatial components $(x, y, z)$. The vector representing the centroid of particle $i$ within $\Omega$ is denoted $\mathbf{p}_{i}$ and the corresponding vectors of particle velocity, acceleration, and force are denoted $\mathbf{v}_{i}, \mathbf{a}_{i}$, and $\mathbf{f}_{i}$ (respectively). The particle mass is $m_{i}$, its radius is $r_{i}$, while the temperature is represented by $T_{i}$, and the heat flux by $q_{i}$. A bond between any pair of particles $i$ and $j$ is represented by the tuple $B_{i j}$.

In the present work we define $\Omega$ to be a regular parallelepiped bounded such that $x_{\min } \leq x \leq x_{\max }, y_{\min } \leq y \leq y_{\max }$, and $0 \leq z \leq z_{\max }$. We consider the gravity to act in the $-z$ direction with a magnitude of $9.81 \mathrm{~m} / \mathrm{s}^{2}$. This domain is demonstrated in Figure 3.

[Figure 3 about here.]

The simulation time variable is $t$, with $t=0$ corresponding to the introduction of the first particles into $\Omega$. The discrete simulation time step is $\Delta t$, and $n_{t}$ is the total number of such time steps in the simulation. For stability, we demand $\Delta t \ll \Delta t_{c r i t}$, which is a critical time step determined from the particle system stiffness. A satisfactory approximation of the critical time step is given by

$$
\Delta t_{c r i t} \simeq \sqrt{\frac{m_{\min }}{k_{n}}}
$$

where $m_{\min }$ is the minimum particle mass and $k_{n}$ is the normal stiffness parameter of the particles from Equation 1. In most cases, satisfactory efficiency can be achieved simply by taking $\Delta t=\Delta t_{\text {crit }} / 10$. More advanced schemes for determining this parameter, such as those given by [58, 59], may be employed in the future.

\subsection{Spatial Searching and Collision Detection}

A cursory analysis of powdered metals available, for example, for SLS additive manufacturing shows that these powders are relatively fine, with particle diameters below 100 microns. As a result very large numbers of particles are 
1 required to model even small additive manufactured objects; on the order of $210^{7}$ particles are required per cubic centimeter of build volume. Additionally, the particle system is highly polydisperse, and contains particles of different sizes. Both of these factors necessitate the use of an efficient collision detection algorithm.

Because of the polydispersity of the particle system, where the ratio of maximum particle size to minimum particle size exceedes ten, we subdivide the 8 collision detection problem into two parts termed 'fine' and 'coarse' detection.

9 The coarse detection uses an octree spatial partitioning scheme similar to that described by [49]. For each particle location $\mathbf{p}_{i}$ the octree is queried to find the 1 indices of the local subset $E_{i}$ of particles

$$
E_{i}=\left\{j:\left\|\mathbf{p}_{i}-\mathbf{p}_{j}\right\| \leq d_{i}\right\}
$$

where $d_{i}$ is the critical collision radius. In general, this is a conservative overestimate of the size of the region which a particle occupies, plus a similar overestimate of the region which another particle occupies. Here, we introduce the assumption that the DEM particles are spherical; based on existing micrographic studies of AM powders [29, 73-75], which show photograpsh of these powders under very high magnification, this is a reasonable approximation. This reduces the calculation of $d_{i}$ to

$$
d_{i}=r_{i}+\max \left(r_{j}, j=1 \ldots N\right)
$$

To avoid excessive computations, the maximal particle radius is precomputed; when particles are added to or removed from the particle system this value is updated.

After coarse collision checking $E_{i}$ contains all the indices of the particles that are tentatively in contact with particle $\mathbf{p}_{i}$. Furthermote, we perform refined collision checking within this vector of potential collisions and we construct the subset $E_{i}^{\prime}$ that contains the indices of the particles that are actually in collision with particle $\mathbf{p}_{i}$, according to

$$
E_{i}^{\prime}=\left\{E_{i}^{j}:\left\|\mathbf{p}_{i}-\mathbf{p}_{E_{i}^{j}}\right\| \leq r_{i}+r_{E_{i}^{j}}\right\} .
$$


1 It is computed by sequentially calculating the Euclidean norm-distance between 2 particles $i$ and all members of $E_{i}$. As per Equation (4), $E_{i}^{j}$ denotes the index of particle $j$ that was found to be in the coarse neihborhood of $i$. In cases where a collision is found, the particle overlap $\delta_{i j}$ between particles $i$ and $j$ is computed for use in the contact model.

\subsection{Contact Modelling}

$7 \quad$ Because of the many collision models available $[52,53]$ we adopt a general 8 approach to the computation of the contact force. Without loss of generality $9 \quad$ we consider the normal and shear forces on particle $i$ generated by contact with 10 particle $j$ to have the form

$$
f_{n_{i j}}=\phi\left(\mathbf{p}_{i}, \mathbf{v}_{i}, \mathbf{v}_{j}, T_{i}, \delta_{i j}\right)
$$

11

$$
f_{s_{i j}}=\zeta\left(\mathbf{p}_{i}, \mathbf{v}_{i}, \mathbf{v}_{j}, T_{i}, f_{n_{i j}}\right)
$$

where $\phi$ and $\zeta$ are arbitrary functions. While this form encompasses many common contact models such as Hertzian contact, or that of Equations (1-2), it does not permit the use of the time-history of the particle positions or other associated quantities. Because of the number of particles in the DEM simulation, storing these time histories is relatively expensive. If a particular application is found which requires these histories, further work will be necessary to determine the most efficient implementation. Once Equations (6-7) have been evaluated for every element of $E_{i}^{\prime}$, the total force on particle $i$ is computed

$$
\mathbf{f}_{i}=\sum_{j=1}^{n_{\epsilon}}\left[f_{n_{i j}}+f_{s_{i j}}\right]+\sum_{j=1}^{n_{B}} f_{B_{i j}}-c_{d} \mathbf{v}_{i}-m_{i} \mathbf{g}
$$

where $n_{\epsilon}$ is the number of contacts, $n_{B}$ is the number of bonds, $c_{d}$ is a viscous drag coefficient and $\mathbf{g}$ is the gravity vector. The computation of the bond forces $f_{B_{i j}}$ is discussed in Section 4.6.

In addition to the generation of mechanical forces, particle collisions also result in the transfer of heat energy. We model this transfer as pure conduction, and neglect radiative or convective effects between particles. Therefore the heat 
1 flux on particle $i$ from particle $j$ is computed by

$$
q_{i j}=k_{t}\left(T_{j}-T_{i}\right)
$$

2 where $k_{t}$ is a heat conduction coefficient which necessarily depends on the physical properties and size of the powder particles, as well as the inter-particle con$\underline{\text { tact cross-sectional area (and thus } \mathbf{f}_{n} \text { ). This coefficient may also be dependent }}$

5 upon on the particle temperature. The total heat flux on particle $i$ is simply

$$
q_{i}=\sum_{j=1}^{n_{\epsilon}} q_{i j}
$$

6

The formulation presented in Equations (8) and (10) neglects the effects of boundary conditions, which are addressed in the next section.

\subsection{Boundary Conditions}

In this work we employ planar spatial boundary conditions. These planar boundary conditions may be infinite, half-infinite, or fully bounded in size. Additionally, these planes may be stationary, or move over time. The particle-plane collision detection of [51] is used. Because of the limited role played by spatial boundary conditions in the AM process we do not imbue them with a sophisticated material model. If particle $i$ impinges on bounding plane $k$ defined by a unit normal vector $\mathbf{n}_{k}$, it experiences a normal to the plane force

$$
\mathbf{f}_{b n_{i, k}}=k_{b}\left(r_{i}-\delta_{b}\right) \mathbf{n}_{k}
$$

where $k_{b}$ is the boundary stiffness parameter and $\delta_{b}$ is the minimal distance between the particle $i$ and the bounding plane $k$. From this normal force, a shear force is also computed by

$$
\mathbf{f}_{b s_{i, k}}=\mu_{b}|| \mathbf{f}_{b n_{i, k}} \| \overline{\mathbf{v}}_{i}
$$

where $\mu_{b}$ is the boundary friction coefficient and $\overline{\mathbf{v}}_{i}$ is the particle velocity projected onto the bounding plane. In addition to these mechanical forces, the planar boundary conditions also participate in heat transfer. If a particle is in contact with a bounding plane, its heat flux is adjusted

$$
q_{i}+=k_{t b}\left(\tau_{e}-T_{i}\right)
$$


1 with $k_{t b}$ corresponding to the head conduction coefficient for the boundary and $\tau_{e}$ is the the 'environment' or process background temperature. Typically, we take $k_{t b}=k_{t}$. For the most part the planar boundary conditions serve to dissipate heat energy, which is introduced into the particle system by a concentrated source. This is discussed in the next section.

\subsection{Energy Application}

The advantageous aspects of additive manufacturing are derived from the highly directed manner in which energy is transferred to the raw feedstock material. In powder-based additive manufacturing systems this is achieved by means of a laser or electron beam, the position and orientation of which is guided by a computer-controlled mechatronic device. The geometric complexity observed in existing additive manufacturing processes dictates that the pattern of heat deposition into the feedstock powder will be highly non-uniform in terms of both the spatial and temporal variables.

In physical additive manufacturing systems, the movement of the heat source that defines the deposition paths, is dictated by a series of numeric control (NC) directives, often collectively known as 'G-code.' While G-code is a specific NC programming language, the term is often used in a general sense to refer to between AM technologies, and between AM hardware manufacturers. In the DEM simulation, this NC code is read and interpreted in order to capture the motion of the laser (or e-beam) heat source. The specifics of this interpretation vary between AM technologies; independent interpreters are needed for specific processes. The output of the interpreter is a function of time which outputs the spatial coordinate of the laser, denoted $\gamma(t)$. This function has a piecewisecontinuous form

$$
\gamma(t)= \begin{cases}\gamma_{0}(t), & 0 \leq t<t_{0} \\ \gamma_{1}(t), & t_{0} \leq t<t_{1} \\ \vdots & \\ \gamma_{n}(t), & t_{n-1} \leq t<t_{n}\end{cases}
$$


1 where each of the $\gamma_{n}$ components corresponds to a straight-line, circular, or 2 elliptical motion of the heat source. During the initialization of the DEM simulation, $\gamma(t)$ is uniformly sampled

$$
P=\{\gamma(0), \gamma(\Delta t), \gamma(2 \Delta t), \ldots, \gamma(n \Delta t)\}
$$

4 For periods of time in which the laser turned off, e.g. when powder is being 5 distributed in SLS processes, $P$ is constructed to return a value which signals 6 the DEM simulation to skip the steps described in the next paragraph.

7 The above procedure is a pre-processing step which is performed before the DEM simulation begins. Therefore, the position of the heat source is known at every time step, this information is contained in $P$. With this information, there are many models which may be employed to compute the heat energy transferred to the particles proximal to this location. Some recent examples of such models are presented in [76]. In our DEM implementation the zone of heat input is bounded by a sphere of finite radius $r_{e}$. At every time step $\left(t=n_{t} \Delta t\right)$, after collision and boundary processing, the distance between each particle and $\gamma_{n_{t}}=\gamma\left(n_{t} \Delta t\right)$ is computed. If this distance is less than $r_{e}$ additional heat flux is applied according to

$$
\mathbf{q}_{i}= \begin{cases}\lambda\left(\left\|\mathbf{p}_{i}-\gamma_{n_{t}}\right\|\right), & 0 \leq\left\|\mathbf{p}_{i}-\gamma_{n_{t}}\right\| \leq r_{e} \\ 0, & \text { otherwise }\end{cases}
$$

17 where $\lambda$ is a symmetric function describing the distribution of heat flux within

the sphere. Typical constructions for $\lambda$ include constant heat flux, or a Gaussian distribution. Figure 4 demonstrates the methodology for energy application in a notional fashion.

[Figure 4 about here.]

\subsection{Sintering and Bond Formation}

From a data-management perspective, the most complex aspect of the DEM simulation is the collection of procedures associated with inter-particle bonding. This is because, in contrast to the constitutive contact laws, the bonds are 
The criterion includes a conditon for sufficient temperature

$$
T_{i} \geq \tau_{s} \text { and } T_{j} \geq \tau_{s}
$$

where $\tau_{s}$ is the critical sintering temperature, a condition for sufficient contact 10 pressure

$$
\left\|\mathbf{f}_{n_{i j}}\right\| \leq f_{\text {crit }}
$$

where $f_{\text {crit }}$ is the critical contact compressive force, and a condition for sufficiently small relative velocity

$$
\mathbf{v}_{i} \cdot \mathbf{v}_{j} \leq v_{\text {crit }}
$$

where $v_{\text {crit }}$ is the critical relative velocity. As a result of this simple pair-wise local bonding a complex network of bonds rapidly arises in the DEM simulation, trailing the path of the directed energy application. A two-dimensional example of this process is shown in Figure 5.

[Figure 5 about here.]

Storing and managing the structure of bonds in the DEM simulation is equivalent to the problem of graph adjacency, as described by [77], with the DEM particles being analogous to graph vertices and inter-particle bonds analogous to graph edges. Due to the size of the DEM particle system, and the sparse localized nature of the bonding process, we employ an adjacency list approach to storing the bonds. The particle centroids $\mathbf{p}_{i}$ are used as a vertex list $V$, which is augmented by an adjacency list $B$. This list is structured such that each element $B_{i}$ is itself a list of vectors. Each of these vectors, denoted $B_{i j}$, 
1 corresponds to the $j^{\text {th }}$ inter-particle bond associated with particle $i$. In the 2 present work we use the form

$$
B_{i j}=\left\{k, \delta_{0}, k_{B}, \varepsilon_{\infty}\right\}
$$

3 where $k$ is the index of the particle with which $i$ is bonded, $\delta_{0}$ is the initial bond

4 length, $k_{B}$ is the bond stiffness, and $\varepsilon_{\infty}$ is the bond failure strain. In general

5 the last two parameters have the form

$$
\begin{aligned}
& k_{B}=\theta\left(T_{i}, T_{k}, \mathbf{f}_{n_{i, k}}\right) \\
& \varepsilon_{\infty}=\eta\left(T_{i}, T_{k}, \mathbf{f}_{n_{i, k}}\right)
\end{aligned}
$$

where $\theta$ and $\eta$ are functions chosen to capture the effects of contact pressure and temperature on the sintering process.

At every time step each bond is evaluated in one of two ways. If $T_{i} \geq \tau_{s}$ then the bonds do not exert a mechanical force on particle $i$. Instead, the bond 11 properties of $B_{i j}$ are recomputed using the conditions of the current time step. ${ }_{12}$ If $T_{i}<\tau_{s}$, then for every bond on particle $i$ exerts a force

$$
f_{B_{i j}}=k_{B}\left(\left\|\mathbf{p}_{i}-\mathbf{p}_{j}\right\|-\delta_{0}\right)
$$

where $k_{B}$ and $\delta_{0}$ are taken from $B_{i j}$. It is important to note that, because different bonds will possess different coefficients, simple bond constitutive behaviors may produce long-range anisotropic responses in the bonded DEM particle system. When a bond reaches the failure strain

$$
\left|\frac{\left\|\mathbf{p}_{i}-\mathbf{p}_{j}\right\|-\delta_{0}}{\delta_{0}}\right|>\varepsilon_{\infty}
$$

it is assumed to fail cleanly and instantaneously. The corresponding entry in $B_{i}$ is deleted and the bond ceases to exist on all subsequent time steps.

\subsection{Time Step Integration}

While a number of different time-step integration schemes have been developed for the DEM [58], in this work we use the common Euler explicit integration method. While Verlet integration is generally superior in DEM applications, we 
1 use the Euler algorithm to demonstrate the functionality of the DEM in the 2 context of additive manufacturing even using a minimal implementation of ba-

3 sic algorithms. At every time step, after the forces and heat fluxes have been

4 calculated in the fashion outlined above, we apply the update scheme expressed

5 by

$$
\begin{aligned}
\mathbf{v}_{i}^{t+\Delta t} & =\mathbf{v}_{i}^{t}+\frac{\mathbf{f}_{i}^{t}}{m_{i}} \Delta t \\
\mathbf{p}_{i}^{t+\Delta t} & =\mathbf{p}_{i}^{t}+\mathbf{v}_{i}^{t} \Delta t
\end{aligned}
$$

7 to update the particle positions and velocities, and

$$
T_{i}^{t+\Delta t}=T_{i}^{t}+\frac{q_{i}^{t}}{m_{i}} \Delta t
$$

8 to update the temperatures.

\section{4.8. Visualization and Post-Processing}

In order to develop, debug, calibrate, and use a DEM simulation it is almost mandatory to provide a visual depiction of the particle system to the programmer or user. This is a demanding task, as a typical additive manufacturing DEM simulation involves millions or more particles. Additionally, using conventional techniques, each particle must be displayed as a set of hundreds or thousands of polygonal faces. Despite the power of modern computer graphics processors, it is not practical to display these DEM systems in this fashion. In order to achieve a "real-time" visualization, where the rendering time $t_{v i s} \ll \Delta t$, we adopt an approach commonly employed in the early days of computer graphics when processing resources were much more scarce.

We utilize an approach known as "billboarding," which is described in depth by [78]. For each particle, a rectangular billboard of two triangles with a shared hypotenuse is computed. The center of this billboard is located at $\mathbf{p}_{i}$, and it is oriented such the billboard is normal to the view vector (the vector from the simulation view point to $\mathbf{p}_{i}$ ). Since the particles are presumed to be spherical, a circle may be drawn on this billboard to emulate a sphere. The area outside of the circle is discarded, so as not to obscure other particle billboards. This 
1 technique enables the display of tens of millions of particles at a frame rate adequate for interactive graphics.

\subsection{Implementation}

4 The procedures outlined in the previous sections were implemented in the $5 \mathrm{C}++$ programming language. The flow of execution in this implementation is 6 shown in Figure 6. Notably, it can be seen that the calculations laid out earlier are well-suited to parallel processing [79]. In this case we utilize the OpenMP shared-memory parallel programming framework [80] to parallelize the innermost program loop which applies the calculations given earlier to each particle. In order to provide a graphical user interface (GUI) we make use of the Qt cross-platform application framework [81]. Qt's signal/slot mechanism is also used for communication between the DEM computation thread and the graphics thread. In addition to the display of the particle system, this framework allows for basic user interaction functions, such as file dialogs, adjusting the simulation view point, and issuing start/stop commands to the particle system simulation.

[Figure 6 about here.]

17 5. Application to Laser Sintering

The formulation given in the previous section is very general, and can be applied to any powder-based additive manufacturing technology. In this section, we demonstrate the steps necessary to apply the methodology to Selective Laser Sintering (SLS). We selected this methodology because of its commercial prominence and because it requires the most sophistication in terms of boundary conditions. There are five major steps which must be taken in order to apply the DEM methodology to the SLS Process:

1. Determination of feedstock powder characteristics and properties.

2. Selection of particle constiutive equations.

3. Selection of DEM parameters.

4. Development of appropriate spatial boundary conditions. 
5. Computation of appropriate energy application G-code.

Each of these steps is given in detail in the following sections.

\subsection{Feedstock Powder Characteristics}

For this application, we chose to study 316L stainless steel, which is widely used in SLS additive manufacturing. It is also beneficial that the mechanical properties of the bulk material are very well understood. Previous work [82] has experimentally measured the size distribution of 316L powders intended for use in AM applications. However, because of the bounded nature of particle size we do not entirely agree with the conclusions of [82], particularly the use of a Gaussian distribution to characterize the particle size variation. We extracted the numeric size data from this earlier work, and re-fit Weibull distributions to characterize the variations in particle shape. These data are shown graphically in Figure 7 and numerically in Table 1. Of these three samples, we selected $S 2$ to govern our DEM particle system. Therefore the particle radii are randomly generated from a Weibull distribution with $k=3.55$ and $\lambda=31.4$.

[Figure 7 about here.]

[Table 1 about here.]

In addition to the particle size distribution, the physical properties of $316 \mathrm{~L}$ stainless steel are also required. In this case we draw all values for physical properties from [83]. From these physical properties, the parameters defined in the previous section may be calculated. Before these calculations can be performed, it is necessary to select the exact form of the constitutive equations governing the particle interactions.

\subsection{Constitutive Equation Selection}

The DEM defined in Section 4 embodies the constitutive response of the particle system as four equations: $\phi, \zeta, \theta$, and $\eta$. These functions capture the normal contact force, shear contact force, bond force, and bond failure strain (respectively). Because the present work is exploratory in nature, and 
1 primarily intended to establish the DEM framework for AM problems in general 2 as opposed to rigorously study one particular material system, we choose simple forms for these constitutive equations. We select

$$
\begin{gathered}
\phi\left(\mathbf{p}_{i}, \mathbf{v}_{i}, \mathbf{v}_{j}, T_{i}, \delta\right)=\phi(\delta)=k_{n} \delta \\
\zeta\left(\mathbf{p}_{i}, \mathbf{v}_{i}, \mathbf{v}_{j}, T_{i}, \mathbf{f}_{\mathbf{n}}\right)=\zeta\left(\mathbf{f}_{\mathbf{n}}\right)=-\mu\left\|\mathbf{f}_{\mathbf{n}}\right\| \frac{\mathbf{v}_{i}-\mathbf{v}_{j}}{\left\|\mathbf{v}_{i}-\mathbf{v}_{j}\right\|}
\end{gathered}
$$

5 to model the particle contacts, as shown in Figure 2. A constant value is chosen

6 for both the particle-particle bond stiffness, and the bond failure strain. The

7 bond stiffness is set equal to the particle contact stiffness,

$$
k_{B}=k_{n}
$$

8 and the bond failure strain is

$$
\varepsilon_{\infty}=0.4
$$

9 At present, we focus mainly on the additive manufacturing process, and we 10 observe that this failure point is never reached by bonds in the particle system.

11 In the future, when the AM components are subjected to mechanical load, this

${ }_{12}$ failure point will be of greater importance. To model the laser heating of the 13 powder bed, we take

$$
\lambda\left(\left\|\mathbf{p}_{i}-\gamma_{n_{t}}\right\|\right)=Q \frac{r_{i}^{3}}{r_{e}^{3}}
$$

${ }_{14}$ Where $\mathrm{Q}$ is the total laser power distributed amongst the particles upon which

15 it impinges in a spatially uniform, volumetrically proportional fashion.

\subsection{Parameter Selection}

The formulation of the previous section involves a number of physical constants, and parameters derived from documented physical constants resources. For completeness, Table 2 enumerates the numeric values for all of the DEM simulation parameters used. Notably, the Hertz-Mindlin model is used to compute the normal stiffness, as detailed by [84], and the thermal conduction coefficient is calculated by

$$
k_{t}=\frac{S \Lambda}{2 \bar{r} c_{p}}
$$


1 where $\mathrm{S}$ is the heat transfer surface area $\pi \bar{r}^{2}, \Lambda$ is the material's heat conductiv-

2 ity, $\bar{r}$ is the average particle radius, and $c_{p}$ is the material's heat capacity. Values

3 for the laser properties were derived from a cursory overview of commercially

4 available SLS systems.

6

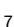

[Table 2 about here.]

The value of the drag coefficient $c_{d}$ is not derived from the physical properties of the additive manufacturing system, environment, or materials. Rather, it is a non-physical parameter used to dissipate kinetic energy imparted to the particles by both gravitation and the scraper boundary condition. Its value was set at the smallest value that produced an equilibrated powder bed within the alloted settling time. We justify this non-physical damping term by noting that the particle system is allowed to equilibrate to a state of effectively zero ki$\underline{\text { netic energy before application of the laser heat source. This implies that the }}$ parameters affecting the free-body motion of the particles will not directly affect the bond-forming sintering process, and will thus not affect the geometry of the bonded particle system. However, this non-physical coefficient should be viewed with caution, and in future implementations constitutive relationships that dissipate energy in a more physically realistic manner should be considered.

\subsection{Mechanical Boundary Conditions}

The purpose of the planar mechanical boundary conditions is to re-create the mechanical processes by which the deposition of uniform layers of feedstock powder is accomplished in a physical SLS machine. We are not concerned with the free granular dynamics of the particles before their entry into the build domain, and therefore do not simulate some aspects of the powder-handling machinery. The methodology employed is to generate a uniform grid of particles (with small perturbations in position), and allow these particles to drop a slight distance into the build domain. This allows the gravitational force to loosely compact the particles into a layer, but does not produce the uniform surface required for additive manufacturing. 

88.

Once the powder particles have been deposited into the build domain, moving boundary conditions are used to compact them into a uniform bed. We experimented with several combinations of boundary conditions for this purpose, such as static or vibrating planes which moved along the $z$-axis in order to achieve compaction. Of these methods, the best performance was achieved using a "scraper" arrangement which closely mimics the physical additive manufacturing process. The scraper boundary conditions are illustrated in Figure

[Figure 8 about here.]

The angled segment of the stationary boundary conditions in Figure 8 is a convenient means to prevent discontinuous application of force to the particle by the boundary conditions. Similarly, the speed of the moving boundary conditions must be restricted in order to prevent destabilization of the explicit time stepping scheme. The results of the simulation are not sensitive to the value chosen for this velocity, in most cases we take the scraper to move at a rate of $10-20 \mathrm{~mm} / \mathrm{s}$.

\subsection{Geometry and Slicing}

In order to simulate the SLS process, it is necessary to convert the digital representation of the object to be produced into a sequence of laser heat-source movements, as described in Section 4. In practice, this is accomplished by a software tool know as a "slicer", which typically takes a triangulated stereolithography (STL) file as input. The topic of slicing algorithms is reviewed by $[85,86]$. Slicing is a demanding geometrical computation with many details and special cases which must be implemented for specific AM technologies, and even specific AM machines. Broadly speaking, the slicer operates by intersecting the STL model with a series of planes in order to define a series of cross sections. For each of these planes, both perimeter and infill trajectories are computed in order to produce a layer.

While several commercial solutions for slicing in the domain of metal SLS additive manufacturing are available, they operate in close connection with the 
1 AM hardware and we were unable to utilize these tools for the present research.

2 Therefore we implemented a simple slicing algorithm which replicated the basic behaviors which we observed in commercial tools. This slicer takes as input the STL model, and parameters for total model height $(h)$, layer thickness $(\Delta h)$, and laser beam radius $\left(r_{e}\right)$. It then intersects the STL model, which consists only of a set of triangular facets, with a series of planes parallel to the $z$-axis spaced at intervals of $\Delta h$. For each layer, this results in a set of closed piecewise-linear curves. For each closed curve, three laser trajectories are computed:

1. A trajectory tracing the closed curve exactly - the "outer perimeter."

2. A trajectory inset from the first by $2 r_{e}$ - the "inner perimeter."

3. A uniform raster pattern with spacing of $2 r_{e}$ which fills the interior of the inner perimeter - the "infill." The raster pattern is rotated by $90^{\circ}$ degrees between layers.

A demonstration of this simple slicer is given in Figure 9. Each of the three paths listed above are uniformly sampled at equal increments corresponding to a given laser velocity $\left(v_{l}\right)$ in order to produce the laser $\mathrm{NC}$ code. Once the laser NC codes for each layer have been produced, they are interleaved with separate commands for the simulation to perform other functions. These include actions such as depositing particles, advancing the scraper boundary conditions, and enforcing a cool-down time after the laser sintering of each layer. This produces a coherent sequence of events which allow the simulated production of the original STL model using the DEM particle system.

[Figure 9 about here.]

\section{Results}

In order to demonstrate the general methodology of Section 4, coupled with the specific methodology for SLS of Section 5, we conducted a pair of test simulations. In both cases the DEM methodology was used to simulate the SLS construction of a different input geometric model. The first test model is the 
well-known "Stanford Bunny," while the second is a small jet turbine blade.

2 In both cases the computing platform employed was a workstation computer, with dual six-core processors operating at $2.0 \mathrm{GHz}$ and $128 \mathrm{~GB}$ memory. We

note that the DEM simulations did not require such a large amount of memory, typically less than one GB was required.

\subsection{Test Case 1: Stanford Bunny}

The first test case utilized a low-polygon interpretation of the well-known "Stanford Bunny" model. The input STL model, and the resultant G-code paths, are shown in Figure 10. The parameters utilized by the slicer are given in Table 3.

[Figure 10 about here.]

[Table 3 about here.]

Several snapshots of the first layer of the DEM simulation can be seen in Figure 11. Snapshots at the completion of several layers, throughout the DEM simulation, are shown in Figure 12. In this figure, the intermediate layers were chosen to show the more irregularly-shaped cross sections of the model while under construction.

[Figure 11 about here.]

[Figure 12 about here.]

A comparison of the final DEM output to the original input model is presented in Figure 13. The output model shown in this figure was computed by performing a Poisson-surface reconstruction [87] of the set of points defined by centroids of all particles which were bonded to at least one other particle, $\left\{i: B_{i} \neq\{\}\right\}$. Inspection of the final portion of Figure 12 and the comparison shown in Figure 13 allows some insight into the behavior of the DEM simulation. It is clear that the DEM simulation of the SLS process recreates the basic geometry of the input model. This is not a perfect translation, and several 
defects can be seen in the output DEM model. In Figure 12 the "stair-step" phenomenon can be clearly seen; this is a well known artifact produced by SLS and other AM systems. Additionally, some loss of definition in the output model is present; for instance the sharp facet edges of the STL model are not seen in the output. This is caused primarily by two factors; the small size of the input model $h=2 \mathrm{~mm}$ and the high rate of heat deposition. The model height is near the lower limit permissible for most commercial systems. This causes particles outside of the domain of the input model to reach temperatures high enough for sintering. Consequently, this promotes the formation of extraneous bumps, as can be seen in several locations on the output model.

[Figure 13 about here.]

\subsection{Test Case 2: Turbine Blade}

While the first test case focused on a well known, but non-functional geometry, the second test case used the geometry of a small jet turbine blade. The input STL model, and the resultant G-code paths, are shown in Figure 14. The parameters utilized by the slicer are given in Table 4 . These parameters are the same as those used in the previous test case, except for the laser power. This has been reduced by a factor of two in order to improve the fidelity of the output DEM model. Additionally, it should be noted that the laser velocity is kept constant, which combined with an input model much larger in size results in a lower power density delivered to the particle system over time.

[Figure 14 about here.]

[Table 4 about here.]

Several snapshots of the one layer of the DEM simulation can be seen in Figure 15. As the first layer is a plain rectangle, the snapshots shown are from a layer where the turbine airfoil is beginning to emerge. Snapshots at the completion of several layers, throughout the DEM simulation, are shown in Figure 16. As before, the intermediate layers were chosen to show interesting non-uniform 
1 sections of the model while under construction. Additionally, the visualization portion of the DEM code was modified so as to not render unbonded particles which were deeply buried in the particle system; this allows a more adequate "live" view of the simulation, which in turn allows the user to monitor developments more clearly.

6

7

10
[Figure 15 about here.]

[Figure 16 about here.]

Finally, a comparison of the DEM output to the original turbine blade model is presented in Figure 17. Here, we see that the thermally induced inaccuracies are smaller, especially examining the lower dovetailed portion of Figure 15 where the stair-step area is located. Some surface irregularity is present, and as before the stair-step effect is clearly present. The broad curved surfaces of the turbine blade exhibit small-scale bumpiness, the source of which is clear when frame three of Figure 15 is examined. The raster heat pattern is producing higher heat densities at the edges of the airfoil surface, which in turn promotes the accumulation of small bumps and irregularities. When the results of Figures 15-17 are viewed together, however, it is clear that the DEM simulation of the SLS process has produced a result of high geometric fidelity.

[Figure 17 about here.]

\subsection{Computational Performance}

The simulation results presented in the previous sections are notable because, despite the modest computing resources employed, they did not require excessive computation time. Table 5 presents the size and computing time required for the test problems.
[Table 5 about here.]
The bulk timing figures of Table 5 can be broken down into the time required to re-build the octree data structure and the time required for the DEM com- putation. In both test cases rebuilding the octree at the beginning of each time 
1 step required approximately $14 \%$ of the total time consumed. Of the remaining

DEM computing time, more that $91 \%$ is spent by the collision-detection algorithm, with the vast majority of this going to the octree search. The remaining

$49 \%$ is dominated by the calculation of particle collision forces and evaluating

5 bond formation. The above mentioned figures refer to a single thread of execu-

6 tion. The collision detection and force/bond formation algorithms are inherently

7 parallelizable and our tests show almost linear scaling with number of threads

8 of execution. It should be noted that the octree rebuild algorithm was not par-

9 allelized.

10 The scaling from the small to the large example is caused by both slowdown

11 in the DEM computation loop (due to the increased number of particles) and

12 due to the longer timespan which must be simulated. The total time period

13 simulated in the turbine blade problem is approximately five times the one re-

14 quired for the Stanford Bunny problem. Given that the ratio of simulation time

15 is roughly 1:5 and the ratio of number of particles is roughly 1:10 the compu-

16 tation time ratio of 1:55 indicates that the DEM implementation is exhibiting

${ }_{17}$ reasonable performance scaling.

\section{Conclusions}

In this work, we have developed a DEM framework for the analysis of particle-based additive manufacturing technologies. This formulation allows us to express the constitutive behaviors of the DEM particles and bonds as a small set of equations and parameters, which may be suitably adapted to a wide range of processes. We have demonstrated the adaptation of this framework by application to a specific additive manufacturing process (SLS), and by further applying this simulated process to a pair of test components. The test results of Section 6 demonstrate that this simulation framework is capable of capturing the spatially and temporally varying distributions of heat and mechanical forces within the additively manufactured object. It is seen that the results of the DEM simulations replicate their input geometries, while also exhibiting 
features which are characteristic of the physical additive manufacturing process which is simulated. Additionally, the timing results of Section 6 show that the additive manufacture of specimens of sufficient size for physical testing is feasible within a modest computational budget.

The present work is exploratory in nature, and our primary intent has been to provide a framework for future detailed studies of additive manufacturing materials, processes, and components. As a result, the models chosen and assumptions made in order to simulate the SLS process and 316L stainless steel feedstock are simplistic in nature. Additionally, there are a wide range of powder-based additive manufacturing feedstock materials and processes, which differ significantly from SLS. Finally, the present work does not encompass the post production loads and fields to which additively manufactured components are subjected. Clearly there are several important development and extensions of the present work which are both valuable and feasible from a technical perspective It is anticipated that the three most important near-term extensions of the present work are:

1. Developing and implementing more realistic particle and bond constitutive models for the $316 \mathrm{~L}$ material system.

2. Developing the boundary condition formulations required for applying multi-physics loads to the bonded particle systems produced by the additive manufacturing process simulation.

3. Developing DEM framework extensions that will enable its implementation for other additive manufacturing technologies such as particle-jet methods.

As part of our planned near-term future work, a Verlet or velocity-Verlet integration algorithm will be employed due to its inherently superior stability and performance. Additionally, a software implementation that utilizes distributed memory parallelization is anticipated to be important for the simulation of larger build volumes.

In addition there are several longer term extensions of the present work 
1 which may be of value, but will require more significant adaptation of the DEM methodology. One such extension is the study of Fused Deposition Modeling (FDM) additive manufacturing processes [88]. These thermoplastic extrusion methods differ substantially from those mentioned in Section 2, and are not based on powder feedstocks. However, because these methods are based on the deposition of heated material which bonds to previous substrate layers, the DEM methodology may be both applicable and computationally feasible. This will require the development of constitutive equations for the DEM which embody the strongly nonlinear responses of polymer materials, which may in turn require substantial revisions to the formalisms defined in Section 4. Because of the relatively low cost and widespread use of FDM in many emerging applications this effort, though involved, it is anticipated that it will offer a great deal of utility.

Perhaps most importantly, the completion of the short term tasks listed above will allow the simulation of loading and failure in additively manufactured test specimens. As discussed earlier, these test specimens will be of dimensions feasible for currently available additive manufacturing systems, loading frames for mechanical testing and 3D metrology systems. This will allow the functional validation of the DEM methodology using a data-driven mixed experimental/numerical technique derived from that of $[89,90]$. This technique, originally employed for the characterization of composite materials, will utilize an 22 automated multiaxial test frame augmeted with a full-field strain imaging sys-

23 tem. This methodology will also be employed to calibrate the parameters of

${ }_{24}$ the DEM models, particularly parameters such as the dissipation term $c_{d}$ of

25 Equation (9). Geometric validation of DEM predictions will be accomplished

26 by utilizing three-dimensional metrology systems that enable geometry recon27 struction from optical or mechanical scanning of AM-built parts. Experimental 28 validation of the DEM methodology in this fashion will allow its use for purposes 29 such as the design, characterization, and certification of additively manufactured 30 components. 
1

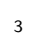

5

\section{Acknowledgements}

The authors acknowledge support for this work by the Office of Naval Research through the Naval Research Laboratory's core funding, as well as the National Research Council's Research Associateship Program.

\section{References}

[1] Ian Gibson, David W Rosen, Brent Stucker, and Others. Additive manufacturing technologies. Springer, 2010.

[2] Samuel H. Huang, Peng Liu, Abhiram Mokasdar, and Liang Hou. Additive manufacturing and its societal impact: A literature review. International Journal of Advanced Manufacturing Technology, 67(5-8):1191-1203, 2013.

[3] John G Michopoulos, Samuel Lamrakos, and Athanasios Iliopoulos. Multiphysics challenges for controlling layered manufacturing processes targeting thermomechanical performance. In ASME 2014 International Design Engineering Technical Conferences $\&$ Computers and Information in Engineering Conference IDETC/CIE 2014, volume I924DV, pages 1-11, Buffalo, New York, August 2014. ASME Press, Order No.: I924DV.

[4] L E Murr, S M Gaytan, F Medina, H Lopez, E Martinez, B I Machado, D H Hernandez, L Martinez, M I Lopez, R B Wicker, and J Bracke. Next-generation biomedical implants using additive manufacturing of complex, cellular and functional mesh arrays. Philosophical transactions. Series A, Mathematical, physical, and engineering sciences, 368(1917):1999-2032, 2010.

[5] Nannan Guo and Ming C. Leu. Additive manufacturing: Technology, applications and research needs. Frontiers of Mechanical Engineering, 8(3):215243, 2013.

[6] I. a. Roberts, C. J. Wang, R. Esterlein, M. Stanford, and D. J. Mynors. A three-dimensional finite element analysis of the temperature field during 
laser melting of metal powders in additive layer manufacturing. International Journal of Machine Tools and Manufacture, 49(12-13):916-923, 2009.

[7] Mahesh Mani, Brandon Lane, Shaw Feng, Shaw Feng, Shawn Moylan, and Ronnie Fesperman. Measurement Science Needs for Real-time Control of Additive Manufacturing Powder Bed Fusion Processes. Technical report, National Institute of Standards and Technology, 2015.

[8] E Oñate and D R J Owen. Particle-Based Methods: Fundamentals and Applications. Computational Methods in Applied Sciences. Springer, 2011.

[9] Paul Francis Jacobs. Rapid prototyping $\&$ manufacturing: fundamentals of stereolithography. Society of Manufacturing Engineers, 1992.

[10] Dietmar W. Hutmacher, Thorsten Schantz, Iwan Zein, Kee Woei Ng, Swee Hin Teoh, and Kim Cheng Tan. Mechanical properties and cell cultural response of polycaprolactone scaffolds designed and fabricated via fused deposition modeling. Journal of Biomedical Materials Research, 55(2):203-216, 2001.

[11] David Bourell, Joseph Beaman, Ming C Leu, and David W Rosen. A brief history of additive manufacturing and the 2009 roadmap for additive manufacturing: looking back and looking ahead. US-Turkey Workshop on Rapid Technologies, pages 5-12, 2009.

[12] Terry Wohlers and Tim Gornet. History of additive manufacturing. Wohlers Report: Additive Manufacturing and $3 D$ Printing State of the Industry Annual Worldwide Progress Report, Wohlers Associates, Fort Collins, CO, 2011.

[13] ASTM Standard 2792, "Standard terminology for additive manufacturing technologies". ASTM International, West Conchocken, PA, 2012. 
[14] Carl R Deckard and Joseph J Beaman. Recent advances in selective laser sintering. In Fourteenth Conference on Production Research and Technology, pages 447-452, 1987.

[15] Ian Gibson and Dongping Shi. Material properties and fabrication parameters in selective laser sintering process. Rapid Prototyping Journal, 3(4):129-136, 1997.

[16] J.P. Kruth, X. Wang, T. Laoui, and L. Froyen. Lasers and materials in selective laser sintering. Assembly Automation, 23(4):357-371, 2003.

[17] JP. Kruth, P Mercelis, J Van Vaerenbergh, L Froyen, and M Rombouts. Binding mechanisms in selective laser sintering and selective laser melting. Rapid Prototyping Journal, 11(1):26-36, 2005.

[18] Mukesh Agarwala, David Bourell, Joseph Beaman, Harris Marcus, and Joel Barlow. Direct selective laser sintering of metals. Rapid Prototyping Journal, 1(1):26-36, 1995.

[19] a. Simchi, F. Petzoldt, and H. Pohl. On the development of direct metal laser sintering for rapid tooling. Journal of Materials Processing Technology, 141(3):319-328, 2003.

[20] a. Simchi. Direct laser sintering of metal powders: Mechanism, kinetics and microstructural features. Materials Science and Engineering A, 428(12):148-158, 2006.

[21] Dongdong Gu and Yifu Shen. Balling phenomena in direct laser sintering of stainless steel powder: Metallurgical mechanisms and control methods. Materials \&3 Design, 30(8):2903-2910, 2009.

[22] Wei-Shao Lin, Thomas L Starr, Bryan T Harris, Amirali Zandinejad, and Dean Morton. Additive manufacturing technology (direct metal laser sintering) as a novel approach to fabricate functionally graded titanium implants: preliminary investigation of fabrication parameters. The International journal of oral \&3 maxillofacial implants, 28(6):1490-1495, 2013. 
[23] Liciane Sabadin Bertol, Wilson Kindlein Júnior, Fabio Pinto Da Silva, and Claus Aumund-Kopp. Medical design: Direct metal laser sintering of Ti6Al-4V. Materials and Design, 31(8):3982-3988, 2010.

[24] Y. Tang, H. T. Loh, Y. S. Wong, J. Y H Fuh, L. Lu, and X. Wang. Direct laser sintering of a copper-based alloy for creating three-dimensional metal parts. Journal of Materials Processing Technology, 140(1-3 SPEC.):368$372,2003$.

[25] O. Scott-Emuakpor, J. Schwartz, T. George, C. Holycross, C. Cross, and J. Slater. Bending fatigue life characterisation of direct metal laser sintering nickel alloy 718. Fatigue \&3 Fracture of Engineering Materials $\& 3$ Structures, pages $n / a-n / a, 2015$.

[26] Diego Manfredi, Flaviana Calignano, Manickavasagam Krishnan, Riccardo Canali, Elisa Paola Ambrosio, and Eleonora Atzeni. From powders to dense metal parts: Characterization of a commercial alsimg alloy processed through direct metal laser sintering. Materials, 6(3):856-869, 2013.

[27] M. Rombouts, J. P. Kruth, L. Froyen, and P. Mercelis. Fundamentals of selective laser melting of alloyed steel powders. CIRP Annals - Manufacturing Technology, 55(1):187-192, 2006.

[28] J. P. Kruth, L. Froyen, J. Van Vaerenbergh, P. Mercelis, M. Rombouts, and B. Lauwers. Selective laser melting of iron-based powder. Journal of Materials Processing Technology, 149(1-3):616-622, 2004.

[29] Chunze Yan, Liang Hao, Ahmed Hussein, and David Raymont. Evaluations of cellular lattice structures manufactured using selective laser melting. International Journal of Machine Tools and Manufacture, 62:32-38, 2012.

[30] Kamran Aamir Mumtaz, Poonjolai Erasenthiran, and Neil Hopkinson. High density selective laser melting of Waspaloy®. Journal of Materials Processing Technology, 195(1-3):77-87, 2008. 
1 [31] Lawrence E. Murr, Sara M. Gaytan, Diana a. Ramirez, Edwin Martinez, 2 Jennifer Hernandez, Krista N. Amato, Patrick W. Shindo, Francisco R. 3 Medina, and Ryan B. Wicker. Metal Fabrication by Additive Manufacturing Using Laser and Electron Beam Melting Technologies. Journal of Materials Science and Technology, 28(1):1-14, 2012.

[32] Denis Cormier, Ola Harrysson, and Harvey West. Characterization of H13 steel produced via electron beam melting. Rapid Prototyping Journal, 10(1):35-41, 2004.

[33] Peter Heinl, Lenka Müller, Carolin Körner, Robert F. Singer, and Frank a. Müller. Cellular Ti-6Al-4V structures with interconnected macro porosity for bone implants fabricated by selective electron beam melting. Acta Biomaterialia, 4(5):1536-1544, 2008.

[34] Edwin Martinez, Lawrence E. Murr, Jennifer Hernandez, Xuemin Pan, Krista Amato, Pedro Frigola, Cesar Terrazas, Sara Gaytan, Emmanuel Rodriguez, Francisco Medina, and Ryan B. Wicker. Microstructures of Niobium Components Fabricated by Electron Beam Melting. Metallography, Microstructure, and Analysis, 2(3):183-189, 2013.

[35] Gary K Lewis and Eric Schlienger. Practical considerations and capabilities for laser assisted direct metal deposition. Materials \& Design, 21(4):417423, 2000.

[36] J. Mazumder, D. Dutta, N. Kikuchi, and a. Ghosh. Closed loop direct metal deposition: Art to Part. Optics and Lasers in Engineering, 34(46):397-414, 2000 .

[37] Amit Bandyopadhyay, B. V. Krishna, Weichang Xue, and Susmita Bose. Application of Laser Engineered Net Shaping (LENS) to manufacture porous and functionally graded structures for load bearing implants. Journal of Materials Science: Materials in Medicine, 20(SUPPL. 1), 2009. 
[38] M L Griffith, D M Keicher, C L Atwood, J A Romero, J E Smugeresky, L D Harwell, and D L Greene. Free form fabrication of metallic components using laser engineered net shaping (LENS). In Proceedings of the Solid Freeform Fabrication Symposium, pages 125-131. University of Texas at Austin Austin, TX, 1996.

[39] Steffen Nowotny, Siegfried Scharek, Eckhard Beyer, and Karl Hermann Richter. Laser beam build-up welding: Precision in repair, surface cladding, and direct 3D metal deposition. Journal of Thermal Spray Technology, 16(3):344-348, 2007.

[40] P. a. Cundall and O. D. L. Strack. A discrete numerical model for granular assemblies. Géotechnique, 29(1):47-65, 1979.

[41] C O'Sullivan. Particulate Discrete Element Modelling: A Geomechanics Perspective. Applied geotechnics. Taylor \& Francis, 2011.

[42] L Jing and O Stephansson. Fundamentals of Discrete Element Methods for Rock Engineering: Theory and Applications: Theory and Applications. Developments in Geotechnical Engineering. Elsevier Science, 2007.

[43] Ferenc Kun and Hans J. Herrmann. A study of fragmentation processes using a discrete element method. Computer Methods in Applied Mechanics and Engineering, 138(1-4):3-18, 1996.

[44] Damien André, Mohamed Jebahi, Ivan Iordanoff, Jean-luc Charles, and Jérôme Néauport. Using the discrete element method to simulate brittle fracture in the indentation of a silica glass with a blunt indenter. Computer Methods in Applied Mechanics and Engineering, 265:136-147, 2013.

[45] L. Vu-Quoc, X. Zhang, and O. R. Walton. A 3-D discrete-element method for dry granular flows of ellipsoidal particles. Computer Methods in Applied Mechanics and Engineering, 187(3-4):483-528, 2000. 
[46] B.K. K Mishra and Raj K. Rajamani. The discrete element method for the $2 \quad$ simulation of ball mills. Applied Mathematical Modelling, 16(11):598-604, $3 \quad 1992$.

4 [47] Gilles Saussine, C. Cholet, P. E. Gautier, F. Dubois, C. Bohatier, and J. J. $5 \quad$ Moreau. Modelling ballast behaviour under dynamic loading. Part 1: A $6 \quad 2 \mathrm{D}$ polygonal discrete element method approach. Computer Methods in $7 \quad$ Applied Mechanics and Engineering, 195(19-22):2841-2859, 2006.

[48] Z.Q. Fang, G.M. Hu, J. Du, Z. Fan, and J. Liu. A Contact detection algorithm for multi-sphere particles by means of two-level-grid-searching in DEM simulations. International Journal for Numerical Methods in Engineering, 2015.

[49] Hiroshi Noborio, Shozo Fukuda, and Suguru Arimoto. Fast interference check method using octree representation. Advanced Robotics, 3(3):193$212,1988$.

[50] J R Williams and R O Connor. Discrete Element Simulation and the Contact Problem. Methods in Engineering, 6(June 1996):279-304, 1999.

[51] C Ericson. Real-Time Collision Detection. Number v. 1 in Morgan Kaufmann series in interactive 3D technology. Taylor \& Francis, 2004.

[52] H. Kruggel-Emden, E. Simsek, S. Rickelt, S. Wirtz, and V. Scherer. Review and extension of normal force models for the Discrete Element Method. Powder Technology, 171(3):157-173, 2007.

[53] Stefan Luding. Cohesive, frictional powders: Contact models for tension. Granular Matter, 10(4):235-246, 2008.

[54] C.L Martin, D Bouvard, and S Shima. Study of particle rearrangement during powder compaction by the Discrete Element Method. Journal of the Mechanics and Physics of Solids, 51(4):667-693, 2003. 
1 [55] Mingjing Jiang, Hai Sui Yu, and Serge Leroueil. A simple and efficient approach to capturing bonding effect in naturally microstructured sands by discrete element method. International Journal for Numerical Methods in Engineering, 69(6):1158-1193, 2007.

[56] Yuanqiang Tan, Dongmin Yang, and Y. Sheng. Study of polycrystalline $\mathrm{Al} 2 \mathrm{O} 3$ machining cracks using discrete element method. International Journal of Machine Tools and Manufacture, 48(9):975-982, 2008.

[57] Yuanqiang Tan, Dongmin Yang, and Yong Sheng. Discrete element method (DEM) modeling of fracture and damage in the machining process of polycrystalline SiC. Journal of the European Ceramic Society, 29(6):1029-1037, 2009.

[58] E. Rougier, a. Munjiza, and N. W M John. Numerical comparison of some explicit time integration schemes used in DEM, FEM/DEM and molecular dynamics. International Journal for Numerical Methods in Engineering, 61(6):856-879, 2004.

[59] K. Samiei, B. Peters, M. Bolten, and a. Frommer. Assessment of the potentials of implicit integration method in discrete element modelling of granular matter. Computers and Chemical Engineering, 49:183-193, 2013.

[60] Loup Verlet. Computer "Experiments" on Classical Fluids. I. Thermodynamical Properties of Lennard-Jones Molecules. Phys. Rev., 159(1):98-103, July 1967.

[61] William C Swope, Hans C Andersen, Peter H Berens, and Kent R Wilson. A computer simulation method for the calculation of equilibrium constants for the formation of physical clusters of molecules: Application to small water clusters. The Journal of Chemical Physics, 76(1), 1982.

[62] K Samiei. Implicit and Explicit Algorithms in Discrete Element Method. Lap Lambert Academic Publishing GmbH KG, 2014. 
2

[63] John Tully. Molecular Dynamics. John Wiley and Sons, Ney York, NY, 2005.

[64] Stefan Luding. From molecular dynamics and particle simulations towards constitutive relations for continuum theory. (Md):1-40.

[65] J J Monaghan. Why Particle Methods Work. SIAM Journal on Scientific and Statistical Computing, 3(4):422-433, 1982.

[66] Joe J Monaghan. Smoothed particle hydrodynamics. Annual review of astronomy and astrophysics, 30:543-574, 1992.

[67] S Li and W K Liu. Meshfree Particle Methods. Springer, 2007.

[68] D Violeau. Fluid Mechanics and the SPH Method: Theory and Applications. OUP Oxford, 2012.

[69] M. B. Liu and G. R. Liu. Smoothed particle hydrodynamics (SPH): An overview and recent developments. Archives of Computational Methods in Engineering, 17(1):25-76, 2010.

[70] Christian Jakob and Heinz Konietzky. Particle Methods: An Overview. PhD thesis, Technical University Bergakademie Freiberg, 2012.

[71] Xiaosong Sun, Mikio Sakai, and Yoshinori Yamada. Three-dimensional simulation of a solid-liquid flow by the DEM-SPH method. Journal of Computational Physics, 248:147-176, 2013.

[72] Jianhua Zhou, Yuwen Zhang, and J. K. Chen. Numerical Simulation of Random Packing of Spherical Particles for Powder-Based Additive Manufacturing. Journal of Manufacturing Science and Engineering, 131(3):031004, 2009.

[73] D. a. Ramirez, L. E. Murr, E. Martinez, D. H. Hernandez, J. L. Martinez, B. I. MacHado, F. Medina, P. Frigola, and R. B. Wicker. Novel precipitatemicrostructural architecture developed in the fabrication of solid copper 
1 components by additive manufacturing using electron beam melting. Acta $2 \quad$ Materialia, 59(10):4088-4099, 2011.

3 [74] X. C. Wang, T. Laoui, J. Bonse, J. P. Kruth, B. Lauwers, and L. Froyen. Direct selective laser sintering of hard metal powders: Experimental study and simulation. International Journal of Advanced Manufacturing Technology, 19(5):351-357, 2002.

[75] Nikolay K. Tolochko, Maxim K. Arshinov, Andrey V. Gusarov, Victor I. Titov, Tahar Laoui, and Ludo Froyen. Mechanisms of selective laser sintering and heat transfer in Ti powder. Rapid Prototyping Journal, 9(5):314$326,2003$.

[76] C D Boley, S A Khairallah, and A M Rubenchik. Calculation of laser absorption by metal powders in additive manufacturing. Applied Optics, 54(9):2477-2482, 2015.

[77] Steven Skiena. Implementing Discrete Mathematics: Combinatorics and Graphy Theory With Mathematica. Basic Books, 1996.

[78] H Nguyen and NVIDIA Corporation. GPU Gems 3. GPU Gems. AddisonWesley, 2008.

[79] John Steuben. Massively Parallel Engineering Simulations on Graphics Processors: Parallelization, Synchronization, and Approximation. $\mathrm{PhD}$ thesis, Colorado School of Mines, 2014.

[80] B Chapman, G Jost, and R van der Pas. Using OpenMP: Portable Shared Memory Parallel Programming. Number v. 10 in Scientific Computation Series. MIT Press, 2008.

[81] S Huang. Qt 5 Blueprints. Packt Publishing, 2015.

[82] A B Spierings, N Herres, and G Levy. Influence of the particle size distribution on surface quality and mechanical properties in AM steel parts. Rapid Prototyping Journal, 17(3):195-202, 2011. 
[83] H E Boyer, T L Gall, and American Society for Metals. Metals Handbook: Desk Edition. American Society for Metals, 1985.

[84] Stef Lommen, Dingena Schott, and Gabriel Lodewijks. DEM speedup: Stiffness effects on behavior of bulk material. Particuology, 12(1):107-112, 2014.

[85] Pu Huang, Charlie C L Wang, and Yong Chen. Algorithms for layered manufacturing in image space. In Advances in Computers and Information in Engineering Research, Volume 1, pages 377-409. ASME Press, 2014.

[86] Pulak Mohan Pandey, N. Venkata Reddy, and Sanjay G. Dhande. Slicing procedures in layered manufacturing: a review. Rapid Prototyping Journal, 9(5):274-288, 2003.

[87] Michael Kazhdan, Matthew Bolitho, and Hugues Hoppe. Poisson surface reconstruction. Eurographics Symposium on Geometry Processing, pages 61-70, 2006.

[88] Iwan Zein, Dietmar W. Hutmacher, Kim Cheng Tan, and Swee Hin Teoh. Fused deposition modeling of novel scaffold architectures for tissue engineering applications. Biomaterials, 23(4):1169-1185, 2002.

[89] J.G. Michopoulos, J.C. Hermanson, A. Iliopoulos, S.G. Lambrakos, and T. Furukawa. Data-driven design optimization for composite material characterization. Journal of Computing and Information Science in Engineering, 11(2), 2011.

[90] J.G. Michopoulos, J. C. Hermanson, and A.P. Iliopoulos. Advances on the constitutive characterization of composites via multiaxial robotic testing and design optimization. In J. G. Michopoulos, D. W. Rosen, C. J. J. Paredis, and J. M. Vance, editors, Advances in Computers and Information in Engineering Research, volume 1, pages 73-95. ASME, New York, 2014. 


\begin{tabular}{cccc}
\hline Sample & Scale $(\lambda)$ & Shape $(k)$ & Median $(\mu m)$ \\
\hline$S 1$ & 17.3 & 2.60 & 15.0 \\
$S 2$ & 31.4 & 3.55 & 28.3 \\
$S 3$ & 41.9 & 2.66 & 36.5 \\
\hline
\end{tabular}

Table 1: Parameters of Weibull distributions of particle diameter for the three samples measured by [84]. 


\begin{tabular}{rccc}
\hline Description & Symbol & Value & Units \\
\hline Universal Properties & & & \\
time step & $\Delta t$ & 0.025 & $\mu s$ \\
gravitation & $g$ & $9.81 * 10^{-6}$ & $\mu \mathrm{m} / \mathrm{s}^{2}$ \\
ambient temperature & $\tau_{e}$ & 100 & ${ }^{\circ} \mathrm{C}$ \\
viscous drag & $c_{d}$ & 0.3 & - \\
\hline Particle Properties & & & \\
density & $\rho$ & $8.0 * 10^{-12}$ & $\mathrm{~g} / \mu \mathrm{m}^{3}$ \\
normal stiffness & $k_{n}$ & $1.2 * 10^{3}$ & $\mathrm{~N} / \mu \mathrm{m}$ \\
friction coefficient & $\mu$ & 0.25 & - \\
thermal coefficient & $k_{t}$ & $8.0 * 10^{-4}$ & $\mathrm{~g} / \mathrm{s}$ \\
\hline Boundary Properties & & & \\
normal stiffness & $k_{b}$ & $2 k_{n}$ & $\mathrm{~N} / \mu \mathrm{m}$ \\
friction coefficient & $\mu_{b}$ & $\mu$ & - \\
thermal coefficient & $k_{t b}$ & $k_{t}$ & $\mathrm{~g} / \mathrm{s}$ \\
\hline Bond Properties & & & \\
stiffness & $k_{B}$ & $k_{n}$ & $\mathrm{~N} / \mu \mathrm{m}$ \\
force criterion & $f_{\text {crit }}$ & 0 & $\mathrm{~N}$ \\
velocity criterion & $v_{c r i t}$ & 5 & $\mu \mathrm{m} / \mathrm{s}$ \\
sintering temperature & $\tau_{s}$ & 1000 & ${ }^{\circ} \mathrm{C}$ \\
\hline Laser Properties & & & \\
laser radius & $r_{e}$ & $50-100$ & $\mu \mathrm{m}$ \\
laser power & $Q$ & $50-400$ & $\mathrm{~W}$ \\
\hline
\end{tabular}

Table 2: Listing of parameters used in the DEM simulation of SLS with 316L stainless steel. 


\begin{tabular}{rccc}
\hline Description & Symbol & Value & Units \\
\hline model height & $h$ & 2 & $\mathrm{~mm}$ \\
layer height & $\Delta h$ & 50 & $\mu \mathrm{m}$ \\
laser power & $Q$ & 200 & $\mathrm{~W}$ \\
laser radius & $r_{e}$ & 50 & $\mu \mathrm{m}$ \\
laser velocity & $v_{l}$ & 1000 & $\mathrm{~mm} / \mathrm{s}$ \\
\hline
\end{tabular}

Table 3: Slicer parameters for the first test problem. 


\begin{tabular}{rccc}
\hline Description & Symbol & Value & Units \\
\hline model height & $h$ & 10 & $\mathrm{~mm}$ \\
layer height & $\Delta h$ & 50 & $\mu \mathrm{m}$ \\
laser power & $Q$ & 100 & $\mathrm{~W}$ \\
laser radius & $r_{e}$ & 50 & $\mu \mathrm{m}$ \\
laser velocity & $v_{l}$ & 1000 & $\mathrm{~mm} / \mathrm{s}$ \\
\hline
\end{tabular}

Table 4: Slicer parameters for the second test problem. 


\begin{tabular}{ccc}
\hline Test & Particles & Time \\
\hline Stanford Bunny & $6.02 * 10^{5}$ & $6.5 \mathrm{hrs}$ \\
Turbine Blade & $6.30 * 10^{6}$ & $345 \mathrm{hrs}$ \\
\hline
\end{tabular}

Table 5: Problem size and timing data for the two test problems. 


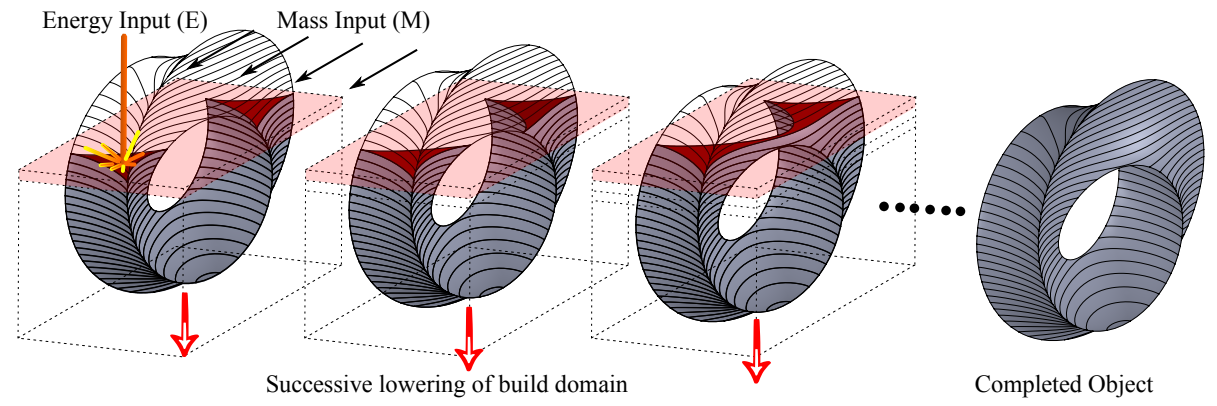

Figure 1: Conceptual overview of powder-based additive manufacturing. 


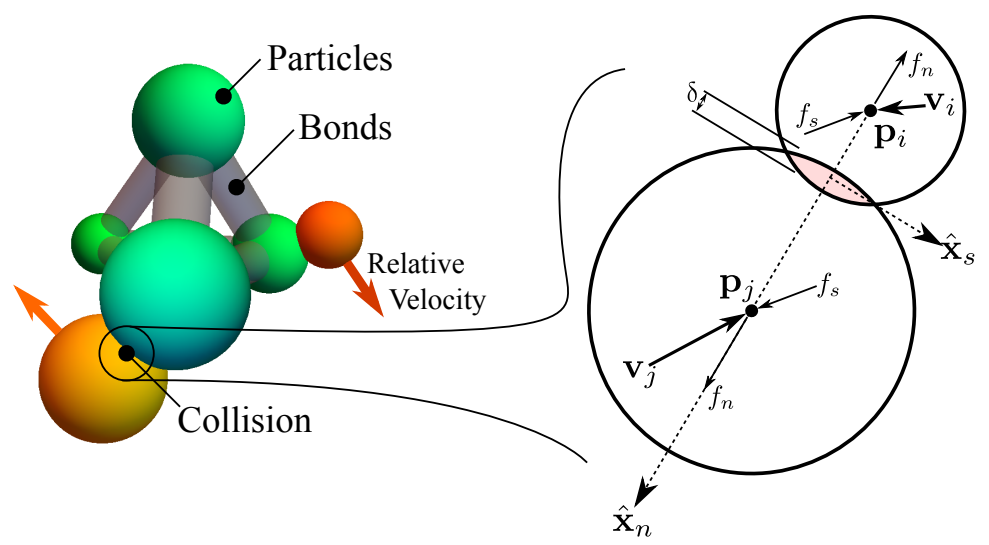

Figure 2: Overview of the Discrete Element Method. At right, the variables associated with the collision mechanics are diagrammed. 


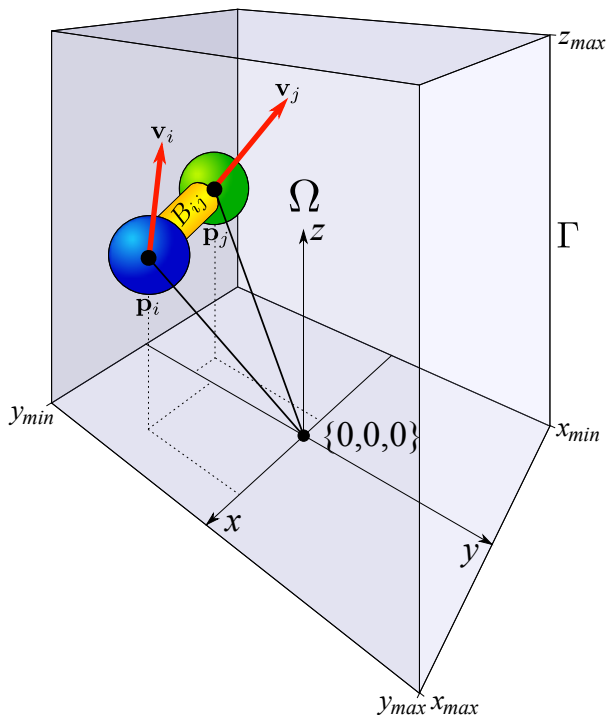

Figure 3: Visualization of the basic DEM domain $\Omega$. Note that the scale of the particles is greatly enlarged. 


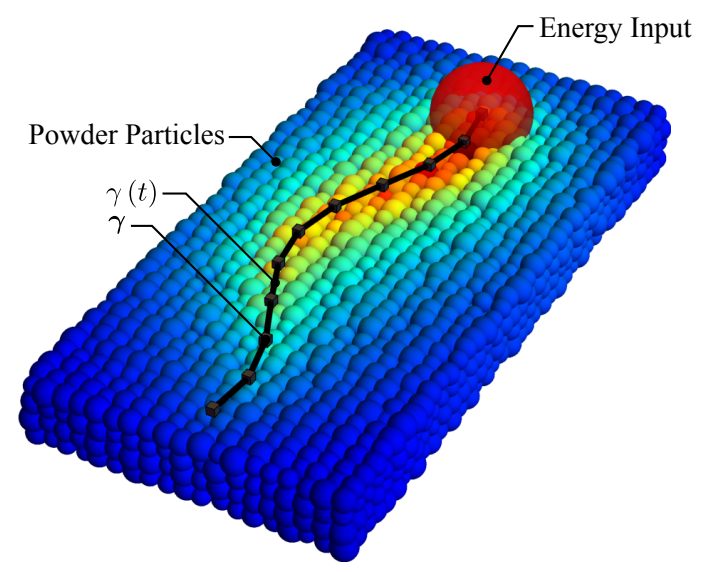

Figure 4: Demonstration of the energy application methodology. Note that particle hue corresponds to the temperature field induced by the application of energy along the path defined by $P$. 


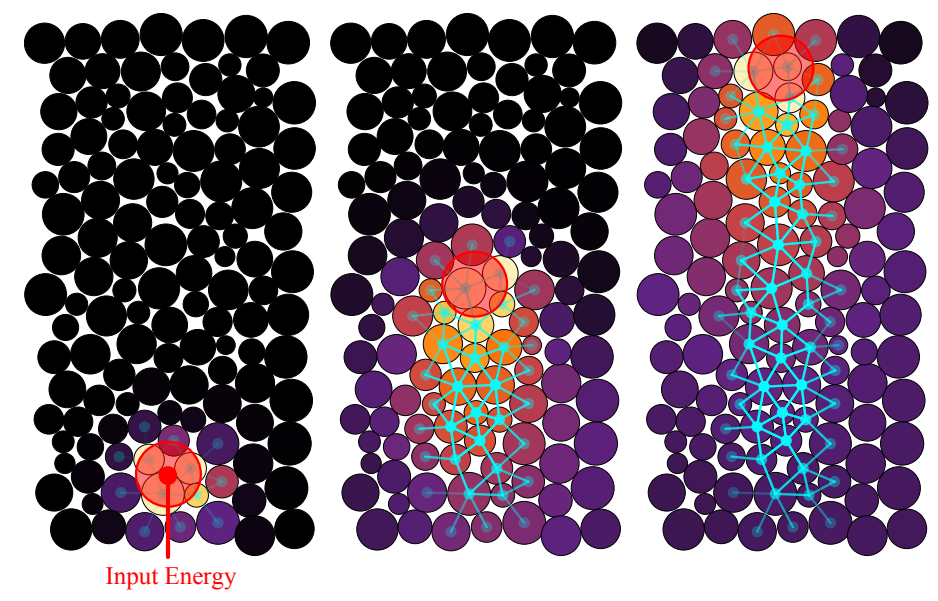

Figure 5: Illustration of bond formation due to a concentrated heat source moving in the upwards direction. Particle hue corresponds to temperature. Bond opacity corresponds to a general notion of bond strength. 


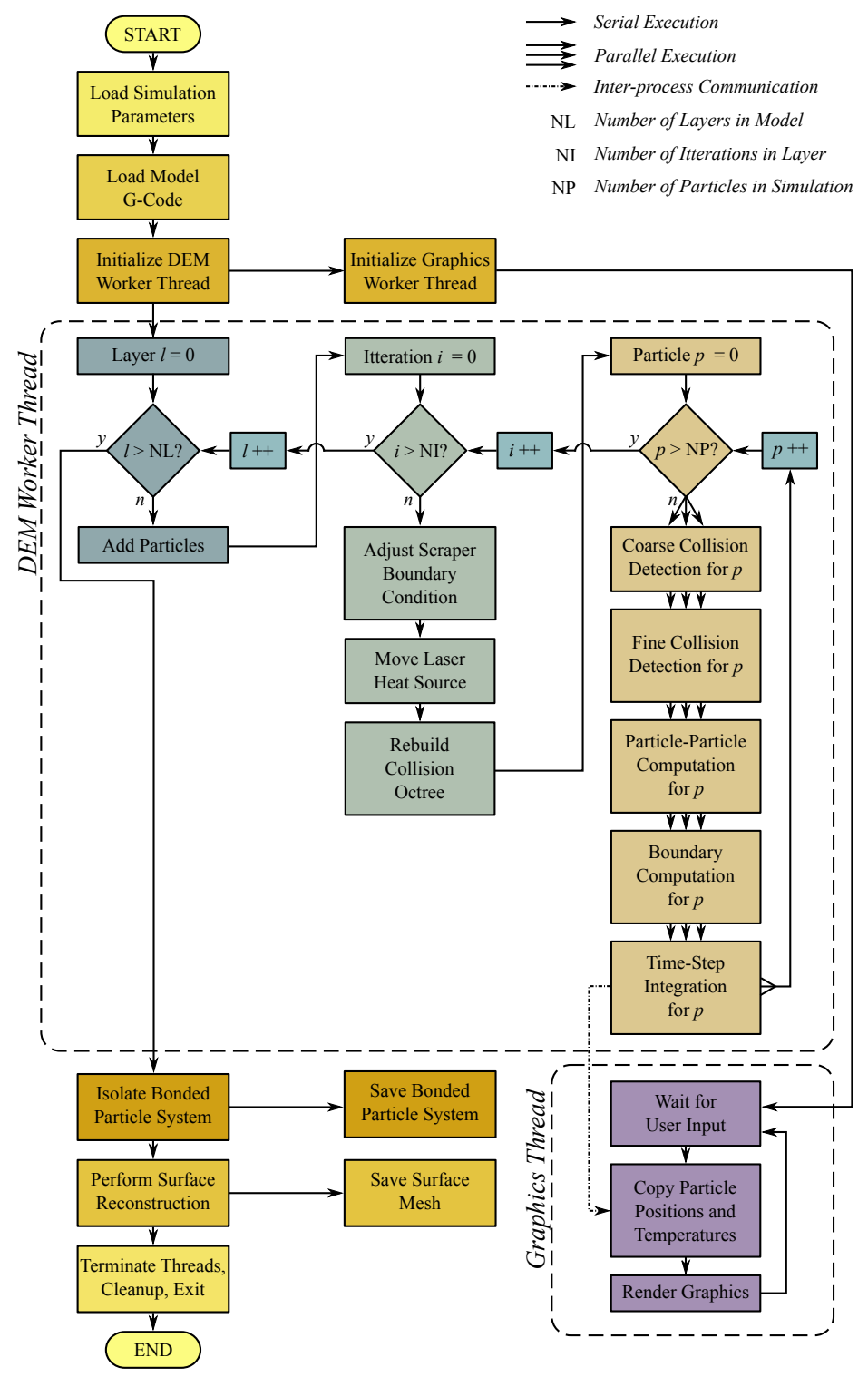

Figure 6: Illustration of the flow of execution in the C++ implementation of the DEM framework. Note the legend at upper right. 


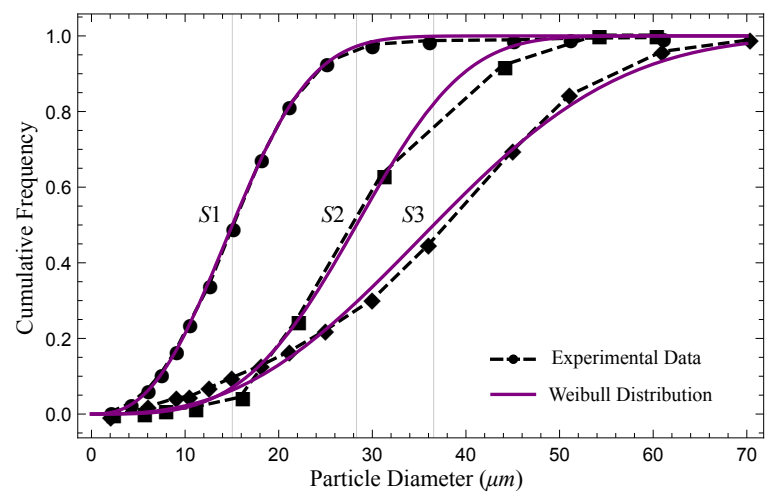

Figure 7: Numeric data and Weibull distribution parameters for three samples of 316L powder intended for use in additive manufacturing. Note the labels $S 1$ through $S 3$, which correspond to the entries in Table 1. 


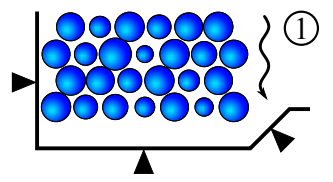

$\mathbf{A}$

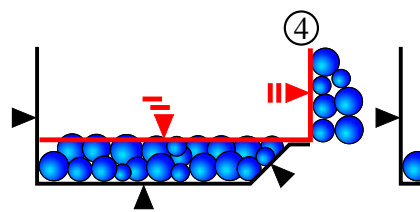

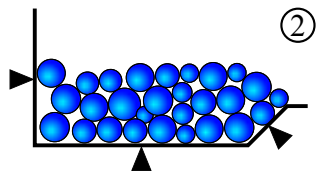

$\boldsymbol{\Delta}$

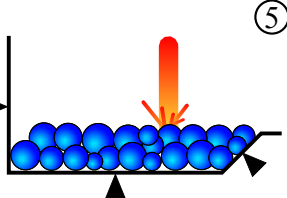

(2)

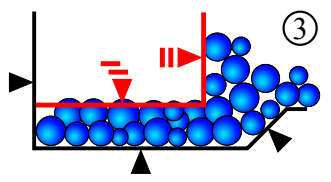

(5)

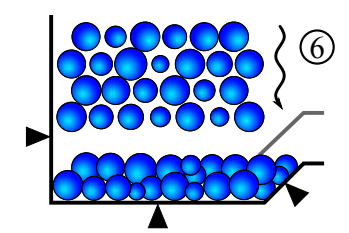

Figure 8: Overview of the scraper boundary conditions for particle compaction. 1) Particles are introduced to the domain in a uniform lattice with small perturbations. 2) Particles are then allowed to settle. 3) A pair of planes travel along the $x$-axis, scraping away excess particles and compacting those that remain into a dense configuration. 4) The scraping planes cross the entirety of the domain; particles which exit are deleted from the simulation. 5) Laser power is applied to sinter the particles. 6) The process begins again, with the boundary conditions suitably adjusted to account for the change in overall height resulting from the buildup of layers. 

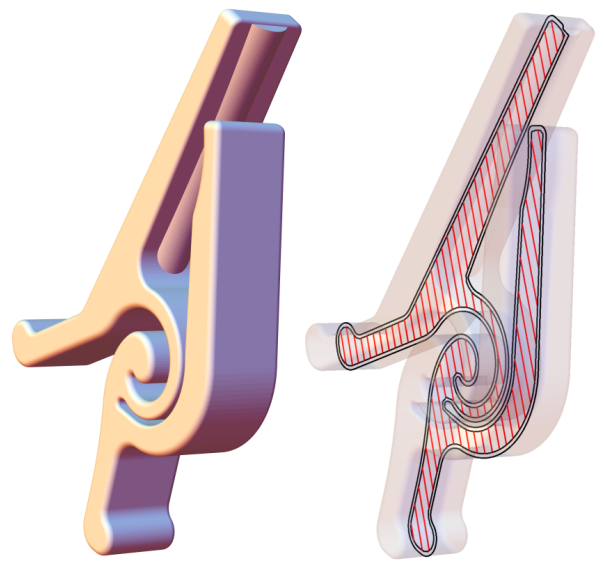

Figure 9: Demonstration of the slicer input STL model (left) and one layer of the output G-code (right). Both the inner and outer perimeters are shown in black, the infill is shown in red. 


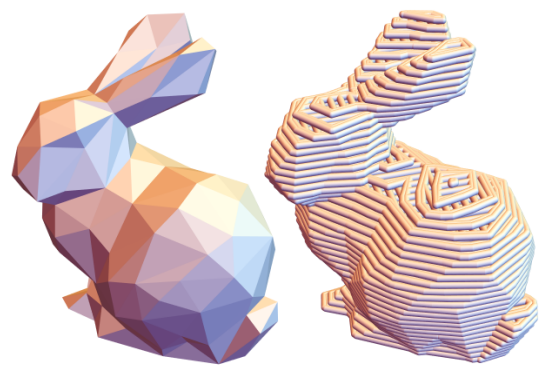

Figure 10: Input STL file (left) and resulting G-code for the first DEM test simulation. 


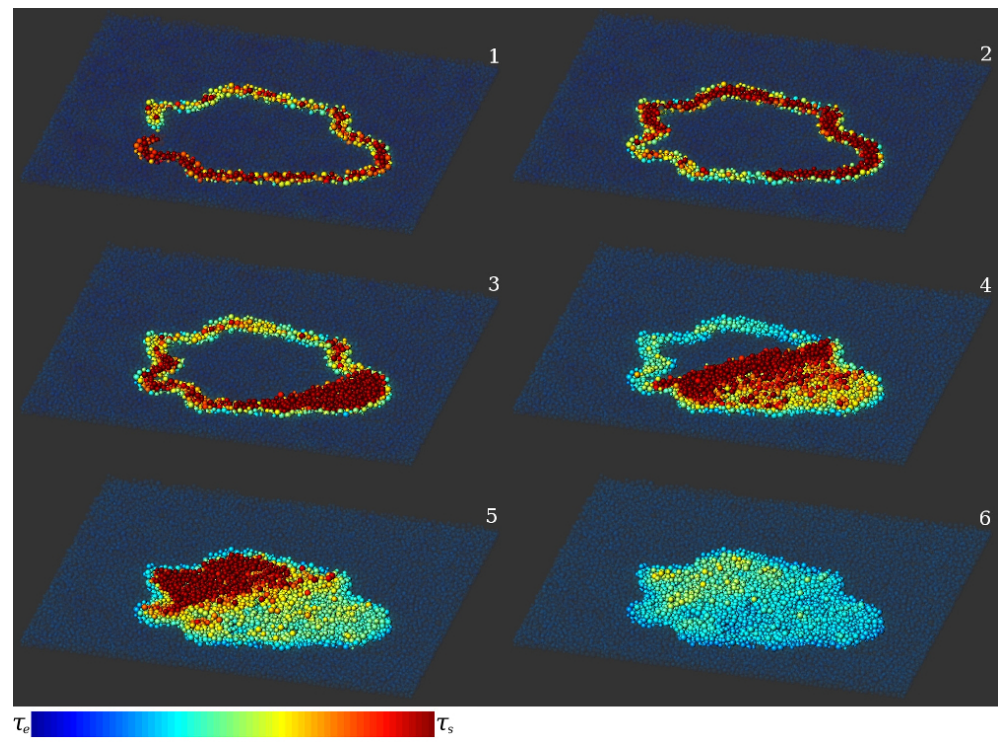

Figure 11: DEM simulation output at six points during the first layer. 1) Near the completion of the outer perimeter. 2) During the second perimeter. 3) Beginning of the infill.) 4) Midpoint of the infill. 5) Infill complete, cooling begins. 6) Cooling time elapsed, immediately before introduction of particles for next layer. 


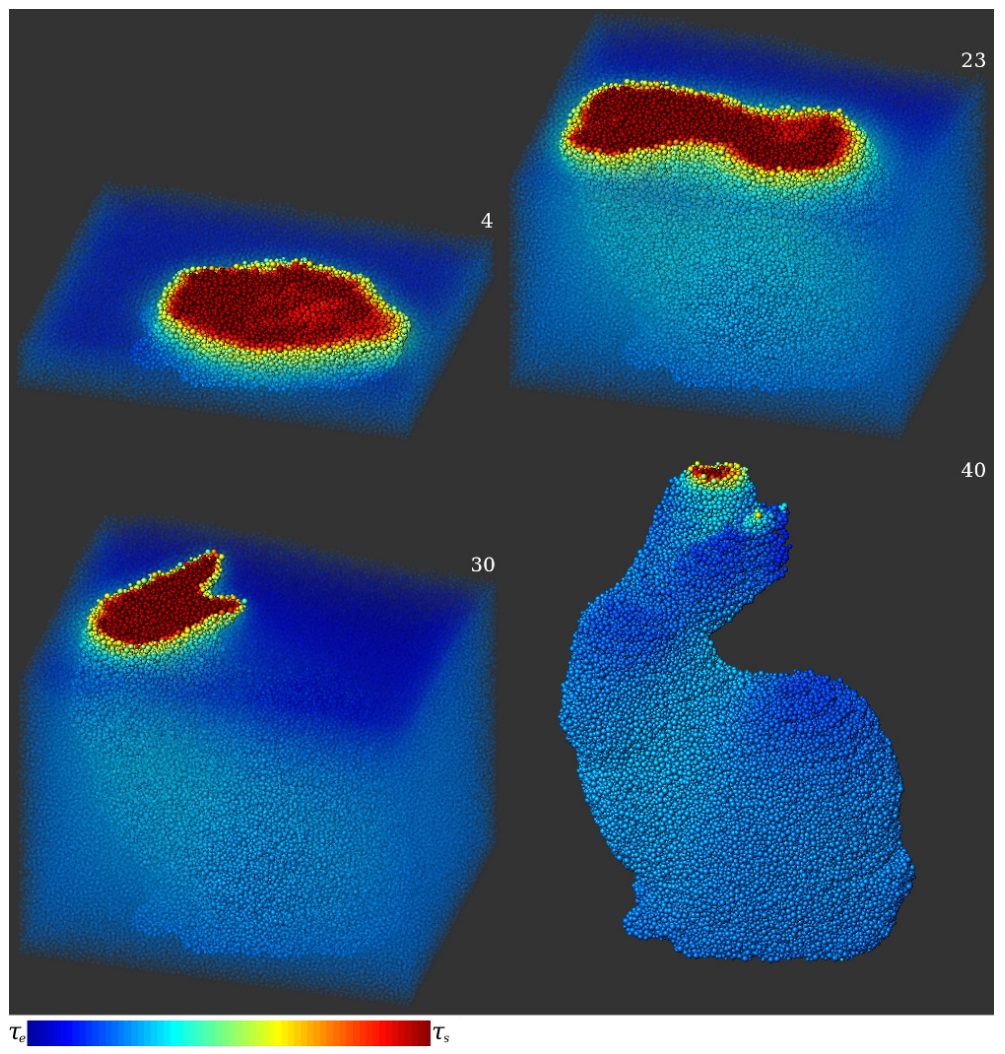

Figure 12: DEM simulation output at the $4^{t h}, 23^{r d}$, and $30^{\text {th }}$ layers, as well as the final output particle system. Particles which have formed no bonds are rendered in a partially transparent fashion in the first three frames, and completely transparent in the final frame. 

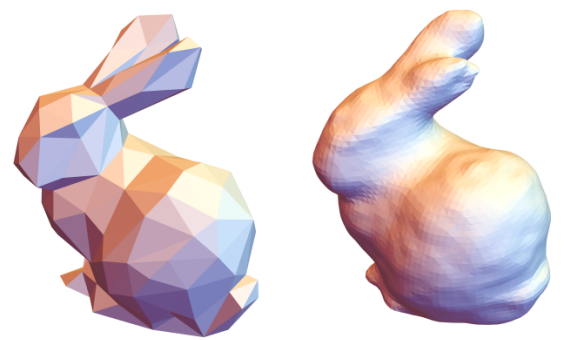

Figure 13: Comparison of DEM input model (left) and output model (right), at the same scale and viewpoint, for the first test problem 


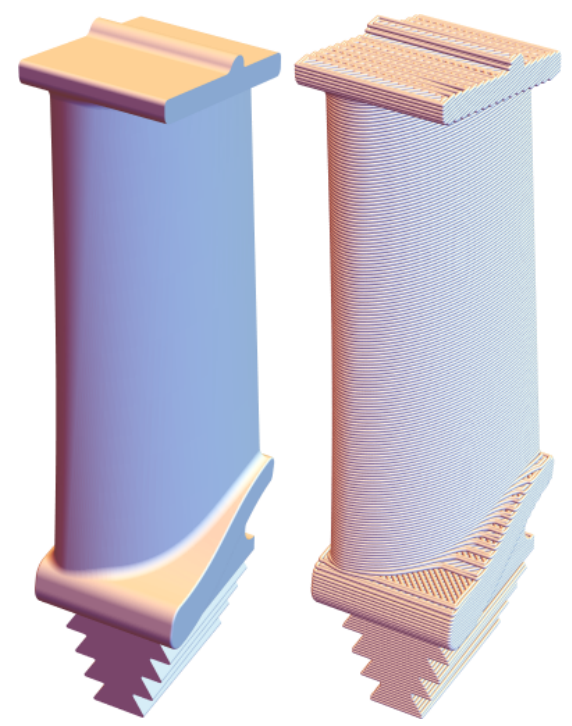

Figure 14: Input STL file (left) and resulting G-code generated paths (right) for the second DEM test simulation. 


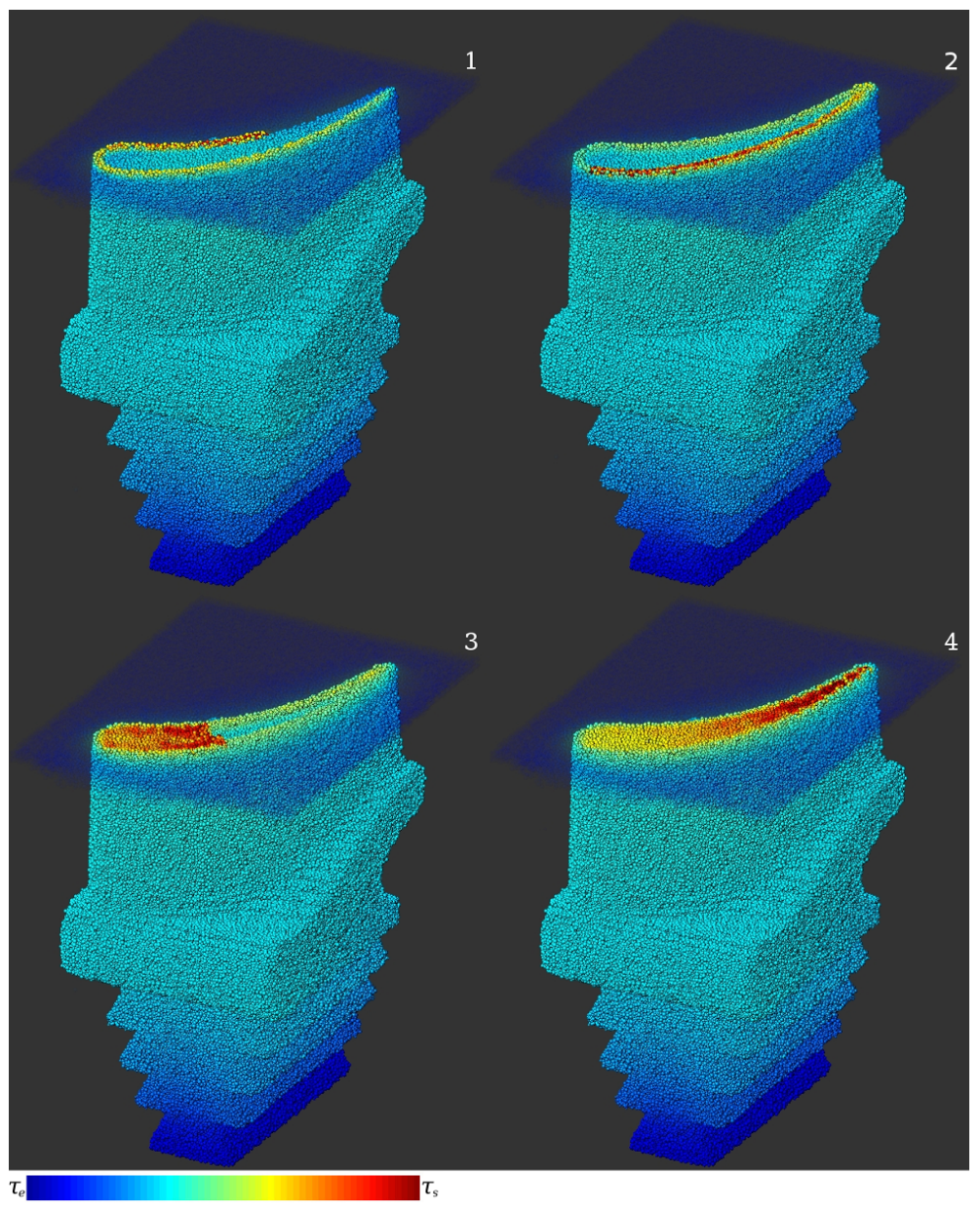

Figure 15: DEM simulation output at four points during the one layer of the second test problem. 1) Near the completion of the outer perimeter. 2) During the second perimeter. 3) Beginning of the infill. 4) Infill complete. 


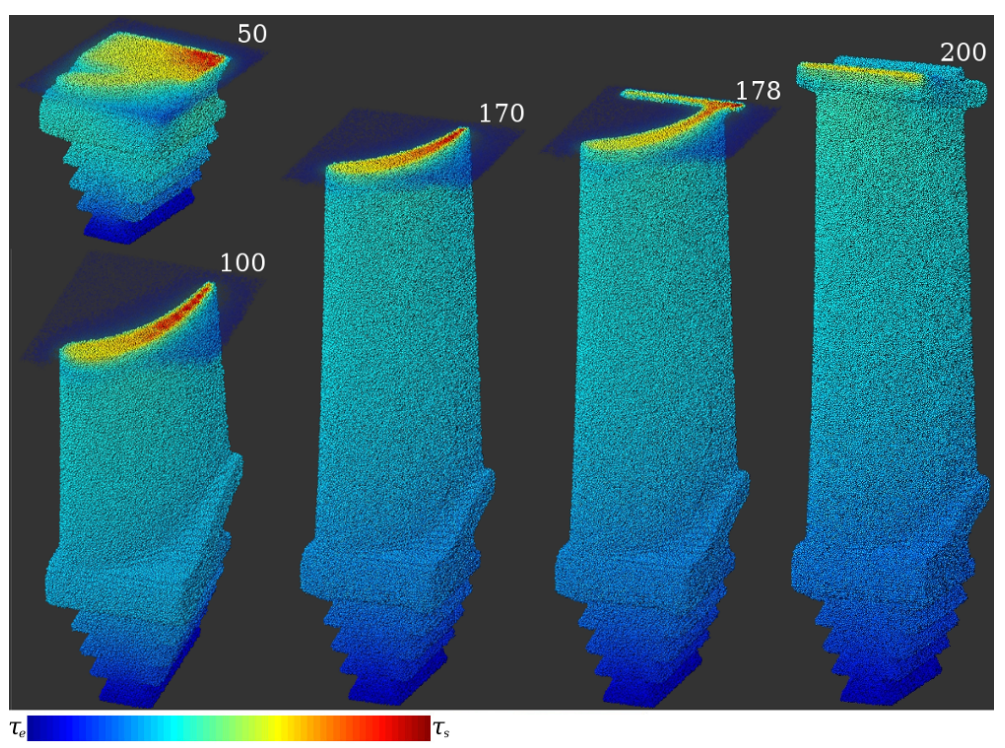

Figure 16: DEM simulation output at the $50^{t h}, 100^{t h}, 170^{t h}$, and $178^{t h}$ layers, as well as the final output particle system. Particles which have formed no bonds are not rendered, unless they are in the top layer. 


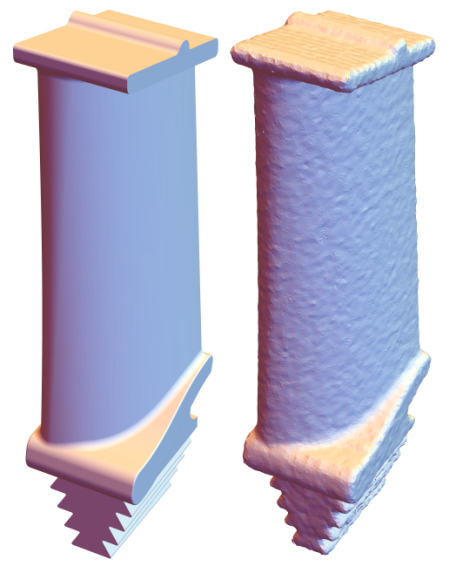

Figure 17: Comparison of DEM input model (left) and output model (right), at the same scale and viewpoint, for the second test problem 